\title{
The cytosolic sensor STING is required for intestinal homeostasis and control of inflammation
}

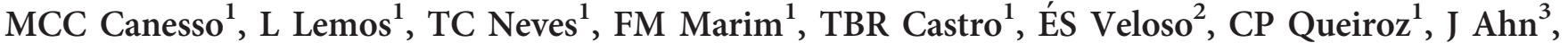 \\ HC Santiago $^{1}$, FS Martins ${ }^{4}$, J Alves-Silva ${ }^{1}$, E Ferreira $^{2}$, DC Cara ${ }^{5}$, AT Vieira ${ }^{1}$, GN Barber ${ }^{3}$, SC Oliveira ${ }^{1}$ and \\ AMC Faria ${ }^{1}$
}

STING (stimulator of interferon genes) is a cytosolic sensor for cyclic dinucleotides and also an adaptor molecule for intracellular DNA receptors. Although STING has important functions in the host defense against pathogens and in autoimmune diseases, its physiological relevance in intestinal homeostasis is largely unknown. In this study, we show that STING ${ }^{-1-}$ mice presented defective protective mechanisms of intestinal mucosa, including decreased number of goblet cells, diminished mucus production, and lower levels of secretory IgA, when compared with wild-type (WT) mice. Fecal content and microbiota DNA could activate STING, indicating a role of this molecule in gut. Microbiota composition was altered in STING ${ }^{-/-}$mice toward a more inflammatory profile, evidencing a reduction in the Allobacolum and Bifidobacterium groups along with increase in Disulfovibrio bacteria. Absence of STING lead to decrease in induced intraepithelial lymphocytes (IEL) and to increase in group 1 innate lymphoid cell (ILC1) as well as ILC3 frequencies and decrease in ILC2 in the colon. Development and function of Foxp3 + and LAP + regulatory Tcells were also compromised in STING ${ }^{-1-}$ mice. Moreover, these mice were highly susceptible to dextran sodium sulfateinduced colitis, T-cell-induced colitis, and enteric Salmonella typhimurium infection when compared with WT animals. Therefore, our results identify an important role of STING in maintaining gut homeostasis and also a protective effect in controlling gut inflammation.

\section{INTRODUCTION}

The large intestine is colonized by trillions of microorganisms that can greatly affect the mucosal immune response. ${ }^{1}$ To maintain intestinal health, the immune system must faithfully respond to antigens from pathogenic microbes while maintaining a state of tolerance to commensals and food antigens that contact it every day. ${ }^{2,3}$ Commensal bacteria have the potential to activate large intestinal epithelial and immune cells and this constitutive activation is critical to maintain intestinal homeostasis. However, defective interactions between the luminal microbiota and intestinal immune system can lead to sustained inflammation and development of chronic diseases, such as inflammatory bowel disease. ${ }^{4,5}$

The intestinal epithelium is renewed at an extraordinary rate, outpacing all other tissues in the body. Cell death, by apoptosis or other mechanisms, are responsible for the renewing of cells; proliferation as well as migration of new cells from precursors induces extrusion of live cells to control epithelial cell numbers. ${ }^{6}$ These events, along with the presence of the microbiota, contribute to release of nucleic acids in the intestine. Extracellular DNA can activate cells of the immune system and a fine balance between pro- and anti-inflammatory mechanisms is required to maintain intestinal homeostasis. ${ }^{7,8}$

Innate immunity has a central role in the homeostasis of intestine and in the pathogenesis of inflammatory bowel disease. A complex network of signaling pathways triggered by molecules derived from commensal microbiota via innate receptors such as Toll-like receptors (TLRs), RIG-I-like receptors, NOD-like receptors (NLRs), and absent in melanoma 2 (AIM2) are employed for the detection of pathogens

${ }^{1}$ Department of Biochemistry and Immunology, Biological Science Institute, Federal University of Minas Gerais, Belo Horizonte, Brazil. ${ }^{2}$ Department of Pathology, Biological Science Institute, Federal University of Minas Gerais, Belo Horizonte, Brazil. ${ }^{3}$ Department of Cell Biology, University of Miami Miller School of Medicine, Miami, Florida, USA. ${ }^{4}$ Department of Microbiology, Biological Science Institute, Federal University of Minas Gerais, Belo Horizonte, Brazil and ${ }^{5}$ Department of Morphology, Biological Science Institute, Federal University of Minas Gerais, Belo Horizonte, Brazil. Correspondence: AMC Faria (anacaetanofaria@gmail.com) 
and for the maintenance of gut integrity in a highly activated environment. ${ }^{7,9}$ Consistently, defects in TLRs (TLR2, TLR4, TLR5, and TLR9), RIG-I-like receptor, NLRs (NOD1, NOD2, NLRP3, and NLRP12) and AIM2 signaling pathways were shown to be involved in the development of colitis and colorectal cancer. ${ }^{8,10}$ However, the precise mechanisms and functions of DNA sensors in intestinal physiology and inflammation have yet to be defined.

STING (stimulator of interferon genes) is a cytoplasmic innate immune sensor for cyclic dinucleotides (CDNs) that also has a role as an adaptor molecule for a number of intracellular DNA receptors. ${ }^{11,12}$ The upstream DNA sensors that signal through STING include cyclic GMP-AMP synthase (cGAS), IFI16, and DDX41, ${ }^{13,14}$ highlighting an important function for STING in controlling multiple DNA recognition pathways. Upon activation, STING recruits TBK1 that phosphorylates IRF3 and nuclear factor (NF)- $\mathrm{kB}$, inducing transcription of type I interferon (IFN) and other co-regulated genes..$^{11-13,15-17}$ Type I IFNs are a widely expressed family of pleiotropic cytokines that exert different functions, including antiviral and antimicrobial activity. Paradoxically, type I IFNs can also suppress immune responses by driving production of antiinflammatory cytokines, and their deregulation contribute to progression of intestinal inflammatory diseases. ${ }^{18-20}$ Since its discovery, STING was shown to contribute to the host defense against viral, bacterial, and eukaryotic pathogens and also to the development of autoimmune disease through its aberrant activation by self DNA. ${ }^{21,22}$ Recent studies have also underscored the role of STING in regulating intestinal inflammation and tumorigenesis in mice. ${ }^{23,24}$ On the other hand, high levels of microbiota-derived DNA and CDNs have been detected in the gut, ${ }^{25,26}$ and these stimuli could contribute to local activation of STING at steady state. Although the role of commensal microbiota in intestinal homeostasis is well documented, ${ }^{27}$ the physiological relevance for STING signaling in this process has not been reported.

In this study, we investigated the physiological function of STING in the gut as well as the impact of its absence in T-cellinduced colitis, in Salmonella infection and in dextran sodium sulfate (DSS)-induced colitis, a model of intestinal inflammatory disease that is associated with epithelial destruction and bacterial translocation. Our results demonstrate that STING ${ }^{-1}$ - mice presented several defects in mechanisms that maintain gut homeostasis both at steady state and under inflammatory conditions.

\section{RESULTS}

\section{Absence of STING resulted in disruption of gut homeostasis}

Intestinal epithelial cells produce antimicrobial effectors that have a central role in shaping the gut microbial community and protecting mucosal tissues from colonization and invasion by commensal microorganisms. ${ }^{8}$ To investigate the potential role of STING in intestine homeostasis, we analyzed histology sections of small intestine and colons from WT and STING ${ }^{-1-}$ mice. First, we observed in hematoxylin and eosin-stained sections that the villi of small intestine in $\mathrm{STING}^{-/-}$mice were elongated and presented less crimps when compared with WT mice (Figure 1a,b). In addition, colon crypts in STING ${ }^{-/-}$ mice were shorter than that in WT mice (Figure 1c,d) resembling the immature phenotype that was already reported for germ-free mice, with altered pattern of microvilli formation and decreased rates of cell turnover. ${ }^{28,29}$ We then examined goblet cell function by using periodic acid-Schiff staining, and STING $^{-/-}$mice displayed decreased numbers of periodic acid-Schiff-positive (goblet) cells per crypt when compared with WT mice (Figure 1d-f). Goblet cells are responsible for mucin production, which is essential to protect the gut mucosa from invasion by colonic bacteria and from other stressors that may cause tissue damage and inflammation. ${ }^{30}$ To further examine whether the lower number of goblet cells in STING $^{-1-}$ mice was associated with mucus production, we stained sections of colon by immunohistochemistry to detect MUC1 and MUC2, which are the major components of the colonic mucus. The number of vesicles positive for MUC1 was smaller in STING ${ }^{-1-}$ mice when compared with WT counterparts (Figure 1g,h). Consistent with that, analysis of the MUC2 staining also showed a decreased frequency of MUC2-expressing cells in STING ${ }^{-1-}$ mice compared with WT mice (Figure 1i $\mathbf{i} \mathbf{j}$ ), indicating that mucus layer production is compromised in the absence of STING.

We next analyzed whether other immune mechanisms involved in mucosa protection were altered in STING ${ }^{-1-}$ mice. Lower levels of secretory IgA (SIgA) were found in the feces of STING ${ }^{-1-}$ mice compared with WT animals (Figure 1k). IgA is essential to gut homeostasis as they reduce the penetration of commensal bacteria in the intestinal epithelium. ${ }^{31}$ Moreover, we assessed the levels of interleukin (IL)- $1 \beta$, which is an important cytokine involved in the maintenance of epithelial barrier, and we observed lower levels of IL-1 $\beta$ in the colon of STING ${ }^{-1-}$ mice (Figure 11). Additionally, mRNA levels of the repair molecule Amphiregulin were also reduced in the colon of STING ${ }^{-1-}$ mice compared with WT mice (Figure 1m) and no difference was found in the levels of $I L-22 b p$ between the studied groups (data not shown). Together, these data indicate that an intact STING signaling is required to maintain several mechanisms of mucosa homeostasis and protection.

As several mechanisms of gut barrier function were defective in STING ${ }^{-/-}$mice, we tested whether intestinal permeability was also compromised in these animals using administration of fluorescein isothiocyanate dextran, but no difference was found between the two groups of mice (data not shown).

\section{Gut microbiota activates STING and induces type I IFN}

STING is known to be activated by cyclic dinucletides that can be produced by many bacteria species, including gut microbiota. To directly assess the involvement of bacteria from gut microbiota in STING activation, we used murine embryonic fibroblasts (MEFs) from WT mice. STING is a transmembrane protein component of endoplasmic reticulum that, when activated, forms an aggregate in the perinuclear region. ${ }^{11}$ MEFs 
a

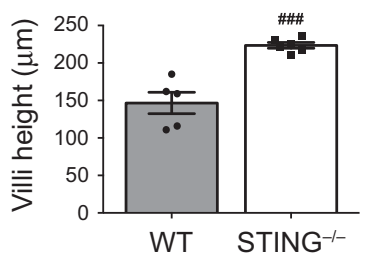

C

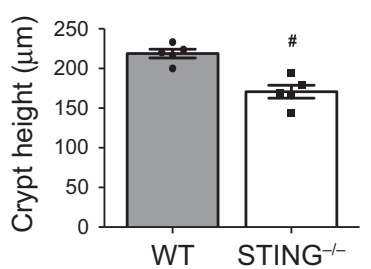

e

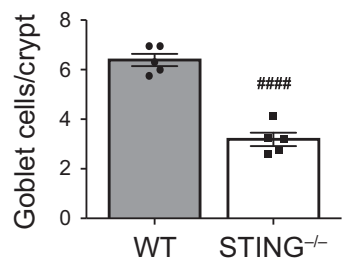

g

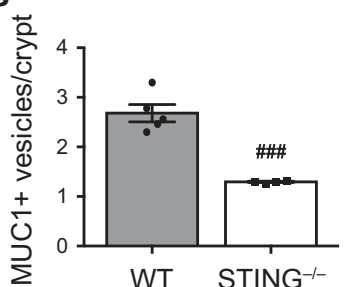

i

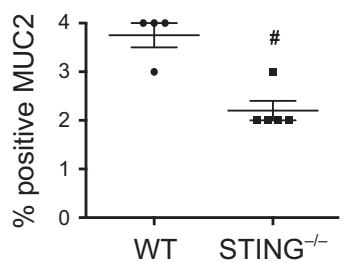

k

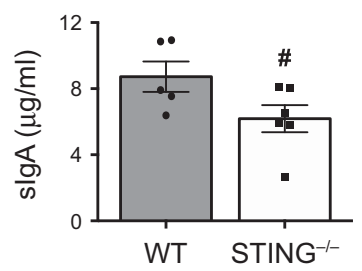

b

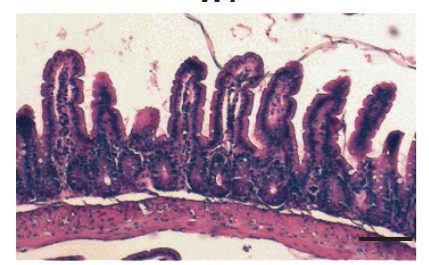

d

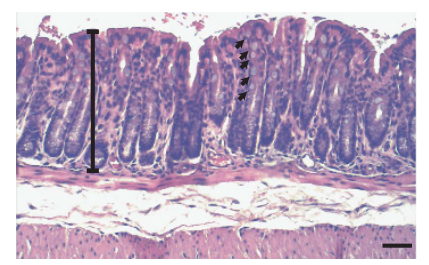

f

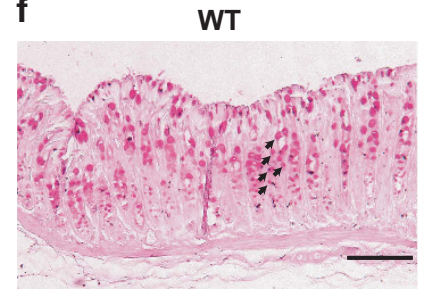

h

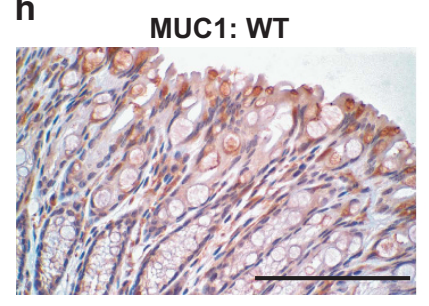

j

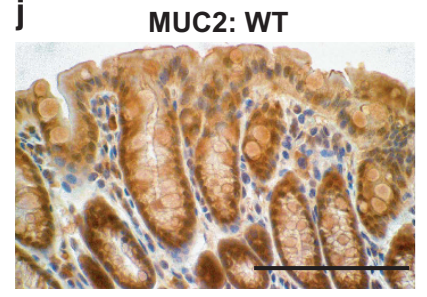

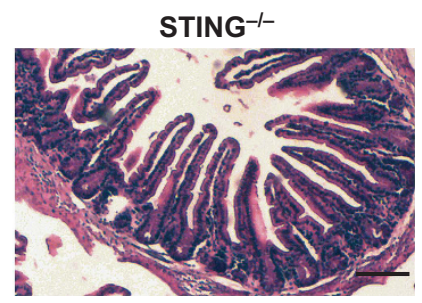

STING ${ }^{-1-}$

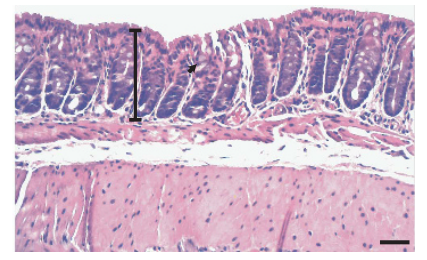

STING ${ }^{-1-}$

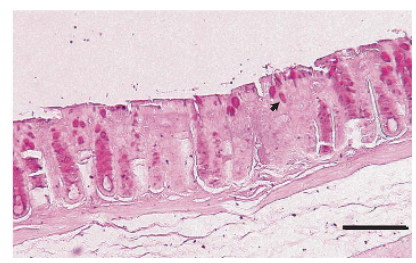

MUC1: STING-I-

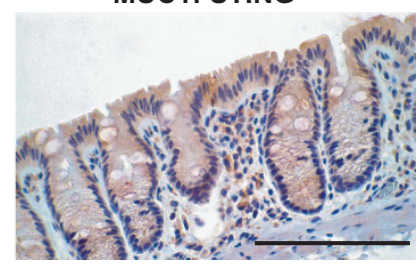

MUC2: STING ${ }^{-1-}$
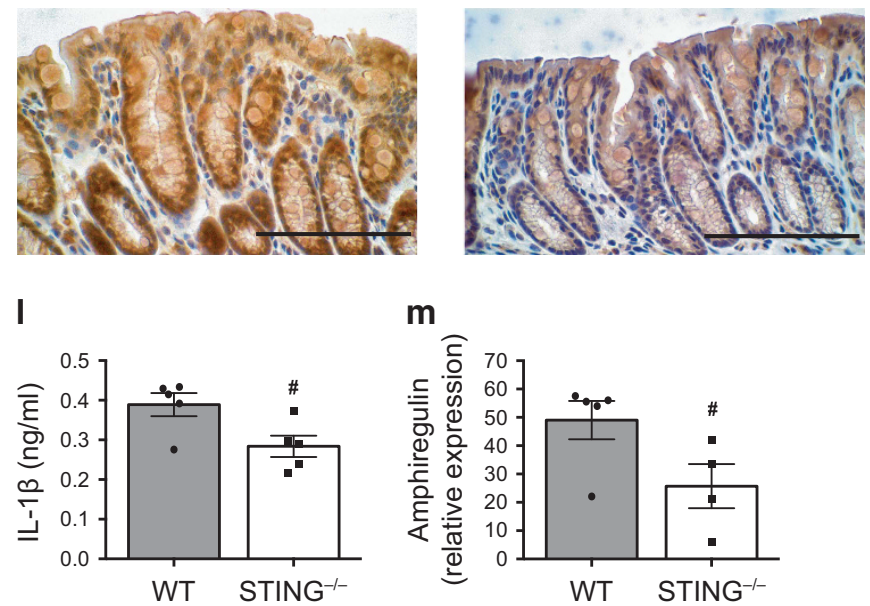

m

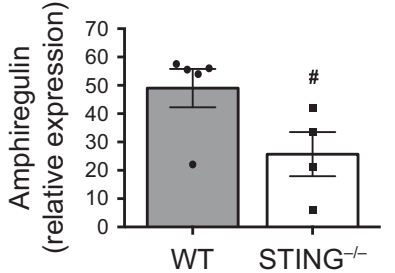

Figure 1 Alterations in mucosa structure in STING ${ }^{-1-}$ mice. Small intestine and colon of wild-type (WT) and STING ${ }^{-/-}$mice were collected for analyses of (a) villi height, (c) crypt height, and (e) the number of goblet cells per crypt. Representative photomicrographies of hematoxylin and eosinstained (b) ileum sections and (d) colon sections from WT and STING ${ }^{-1-}$ mice evidencing villi and crypt height, respectively. Representative photomicrographies of periodic acid-Schiff-stained (f) colon sections from WT and STING ${ }^{-1}$ - mice evidencing goblet cells (arrows). Quantification of (g) MUC1 and (i) MUC2 in the colons of WT and STING ${ }^{-1-}$ mice. Representative photomicrographies of (h) MUC1 and (j) MUC2 immunohistochemical colon sections. (k) Secretory IgA (SIgA) levels in feces and (I) interleukin (IL)-1 $\beta$ levels in the colons of WT and STING ${ }^{-1-}$ mice. (m) Quantitative reverse transcriptase-PCR analysis of Amphiregulin mRNA in the colons from WT or STING ${ }^{-1}$ mice. Bars represent $100 \mu \mathrm{m}$. Data represent two independent

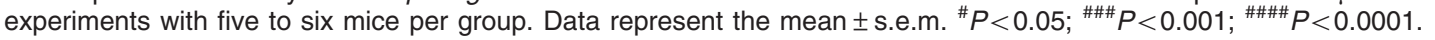


were stimulated for $4 \mathrm{~h}$ with STING-activating dsDNA90 (positive control), fecal content, or microbiota DNA purified from feces and the cells were analyzed by confocal microscopy. In the absence of stimuli, STING was sparse in the cytoplasm. However, when MEFs were stimulated with dsDNA90, fecal content, or DNA purified from the feces, STING migrated and formed a punctual aggregate in the perinuclear region of the cell (Figure 2a). This result suggests that gut commensal microbiota can activate STING.

STING is known to be important for type I IFN induction. ${ }^{11,15,16}$ Indeed, we found significantly reduced levels of IFN$\beta$ mRNA in the colon of STING ${ }^{-1-}$ when compared with WT mice (Figure 2b). Consistent with a role for microbiota in STING activation, WT mice that were treated with a broad spectrum antibiotics showed similar levels of IFN- $\beta$ expression compared with STING ${ }^{-1-}$ mice (Figure 2c). We also found reduced IFN- $\beta$ mRNA levels in the colon of germ-free mice when compared with their WT counterparts (see Supplementary Figure S1 online). Moreover, mono-association of germ-free mice with the commensal bacterium Bacteroides thetaiomicron partially rescued IFN- $\beta$ expression (see Supplementary Figure S1), indicating a role for gut microbiota in type I IFN via STING signaling.

To further demonstrate that gut microbiota activates STING, we cultured lamina propria cells from WT and STING ${ }^{-1-}$ mice and stimulated them for $16 \mathrm{~h}$ with either dsDNA90 or microbiota DNA purified from feces and measured IFN- $\beta$ expression. As expected, colon lamina propria cells from WT mice expressed high levels of IFN- $\beta$ mRNA when stimulated with dsDNA90 and no IFN- $\beta$ expression was observed in $\mathrm{STING}^{-/-}$mice upon the same stimuli (Figure 2d). Moreover, microbiota DNA also induced type I IFN in WT mice lamina propria cells, whereas no expression was found in the absence of STING. Together these data suggest that the microbiota is indeed involved in the production of type I IFN in a STING-dependent pathway.

It has been described that microbiota and immune cells in the gut regulate each other. There are reports, for instance, on the role of microbiota in SIgA production ${ }^{32,33}$ and, reciprocally, on the impact of SIgA in the microbiota composition. ${ }^{34,35}$ Therefore, we next assessed whether the absence of STING signaling by gut microbiota would interfere with its composition. The beta diversity was assessed by the principal coordinate analysis of weighted (Figure 2f) and unweighted (Figure 2g) Unifrac distances. In both metrics, WT and STING ${ }^{-1-}$ mice groups clustered separately and showed to be significantly different by means of the Adonis statistical test (unweighted: $P=0.015$, weighted: $P=0.01$ ). There was a greater fecal output in the Proteobacteria and a reduction in the Actinobacteria phylum in the feces from STING ${ }^{-1-}$ mice, but the most prominent gut microbiota differences between WT and STING $^{-1-}$ mice were observed within specific phyla (Figure 2e,h,i). An overgrowth of Desulfovibrio genus (Proteobacteria phylum), which is related with development of gut inflammation, and a decrease in symbiotic commensal organisms such as Bifidobacterium (Actinobacteria phylum) and Allobaculum (Firmicutes phylum), shown to display antiinflammatory effects, were detected in $\mathrm{STING}^{-1-}$ mice (Figure 2h,i). ${ }^{36-38}$ Significant differences in relative abundance of these bacteria populations within specific phyla between STING $^{-1-}$ and WT mice were confirmed by DESeq2 analysis.

\section{Frequencies of IEL and ILC subsets were altered in the colon of STING ${ }^{-I-}$ mice}

To ensure intestinal homeostasis, local immune cells must be able to respond to pathogen antigens quickly while maintaining the state of tolerance to commensals. We assessed next whether the absence of STING influenced in intraepithelial lymphocyte (IEL) populations (Figure 3a shows gate strategy). Strikingly, the frequency of natural IEL $(\mathrm{CD} 45+\mathrm{TCR} \gamma \delta+)$ was much higher in STING ${ }^{-1-}$ mice in comparison with that in WT mice (Figure 3b). At the same time, the frequency of both subsets of induced IELs (CD45+ TCR $\alpha \beta+\mathrm{CD} 4$ $\mathrm{CD} 8 \alpha \beta+, \quad \mathrm{CD} 45+\mathrm{TCR} \alpha \beta+\mathrm{CD} 4-\mathrm{CD} 8 \alpha \alpha+) \quad$ was significantly reduced in STING ${ }^{-1-}$ mice compared with WT (Figure 3c-f). This IEL phenotype observed in STING ${ }^{-1-}$ mice resembles what is observed in germ-free mice featuring an immature development of gut immunity. ${ }^{39,40}$

We then examine whether STING would also interfere in the colon lamina propria cells. There was no difference in the frequency of T helper type 1 (Th1), Th2, and Th17 cells in the colon lamina propria of STING ${ }^{-1-}$ mice when compared with WT animals (see Supplementary Figure S2b,c,d), suggesting that Th cells were not affected by the absence of STING. Next, we analyzed innate lymphoid cells (ILCs) since these cells are substantially enriched in mucosal surfaces and seem to be particularly important regulators of epithelial barriers in the intestine. ${ }^{41,42}$ Interestingly, the frequency of ILC1 (Figure 3g,h) and ILC3 (Figure 3g,j) was increased in the colonic lamina propria of STING ${ }^{-1-}$ mice compared with WT (Supplementary Figure S3 shows gating strategy). Moreover, the frequency of ILC2 (Figure 3g,i) was reduced in the colonic lamina propria of STING ${ }^{-1-}$ mice, a result that parallels with the reduced levels of IL-4 and IL-5 (Figure 3k) and increased levels of IL-17 (Figure 31) in the colon of these mice. This data suggests that lack of STING results in an imbalance in the frequency of ILCs, which might be harmful to gut homeostasis.

\section{Lack of STING signaling reduced the frequency and function of regulatory $T$ cells}

Regulatory $\mathrm{T}$ (Treg) cells expressing Foxp3 or latencyassociated peptide (LAP) mediate suppression of a variety of immune cells and actively prevent inflammatory bowel diseases and food allergies. ${ }^{43,44}$ It has been previously shown that type I IFNs regulates Treg accumulation in the intestine and maintains their stability. The absence of IFNAR (IFNalpha receptor) signaling on innate immune cells results in poor expansion and maintenance of Foxp3 + Tregs in the intestine. ${ }^{20,45,46}$ Given that STING signaling induces the production of type I IFNs, we analyzed whether the absence of STING would interfere with the development and function of Treg cells in gut mucosa. STING ${ }^{-1-}$ mice had lower frequency of $\mathrm{CD} 4+\mathrm{LAP}+$ Treg cells in colonic lamina propria and lower 
a
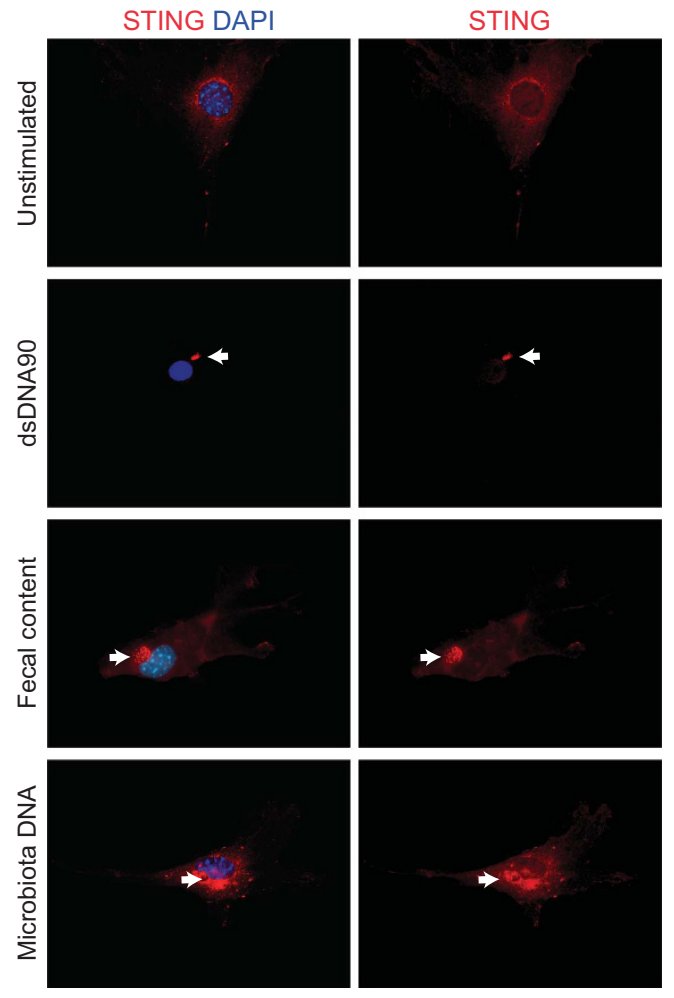

b
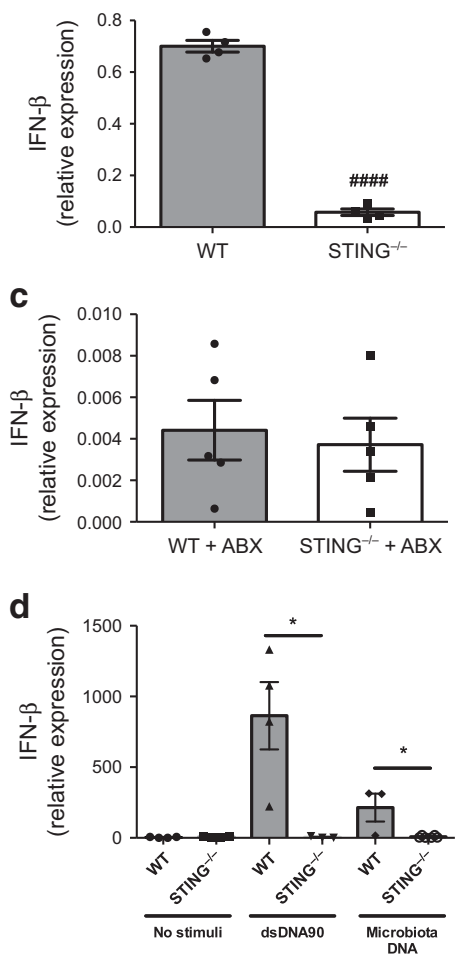

e

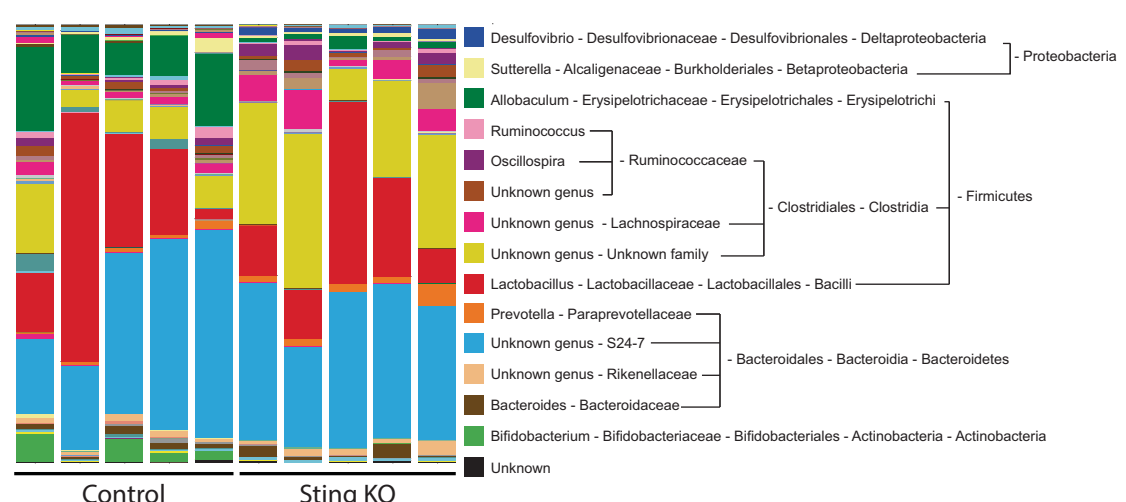

f

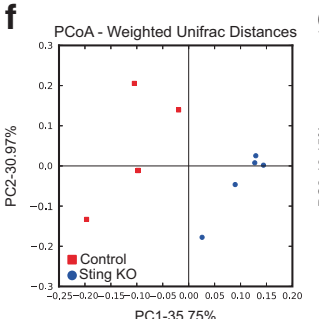

Sting $\mathrm{KO}$

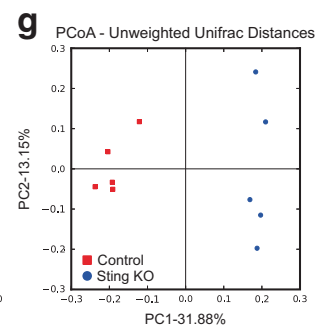

$\mathbf{h}$

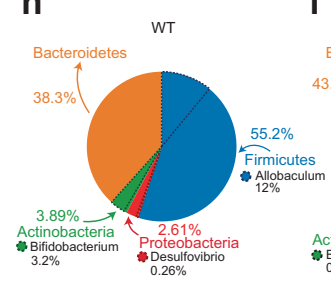

i
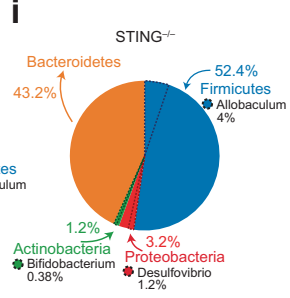

Figure 2 Activation of STING (stimulator of interferon genes) by commensals. (a) Confocal microscopy of wild-type (WT) murine embryonic fibroblasts (MEFs) stained with anti-STING and DAPI (4,6-diamidino-2-phenylindole), unstimulated or stimulated for $4 \mathrm{~h}$ with STING-activating dsDNA (dsDNA90 base pairs), fecal content, or microbiota DNA purified from feces from WT mice. MEFs from STING ${ }^{-1-}$ mice did not stain with anti-STING (data not shown). Arrows highlight STING punctual aggregation. (b) Quantitative reverse transcriptase-PCR (qRT-PCR) analysis of interferon (IFN)- $\beta$ mRNA in the colons from WT or STING ${ }^{-1}$ - mice. Data represent two independent experiments with four mice per group. (c) qRT-PCR analysis of IFN- $\beta$ mRNA in the colons from WT or STING ${ }^{-1-}$ mice treated with a broad spectrum of antibiotics. (d) qRT-PCR analysis of IFN- $\beta$ mRNA of WT and STING ${ }^{-1-}$ mice lamina propria cell culture stimulated with STING-activating dsDNA or microbiota DNA purified from feces for $16 \mathrm{~h}$. (e) Distribution of operational taxonomic units in feces from WT and STING ${ }^{-1-}$ mice. Assessment of structure of microbial communities by (f) weighted and $(\mathbf{g})$ unweighted UniFrac principal coordinate analyses (PCoA) plots are presented for gut bacteria sequenced. Distribution of bacteria phyla in feces from (h) WT and (i) STING ${ }^{-1-}$ mice evidencing main differences within each phylum. Data represent two independent experiments with three to five mice per group. Data

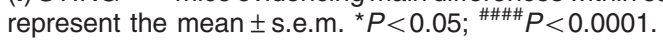


a

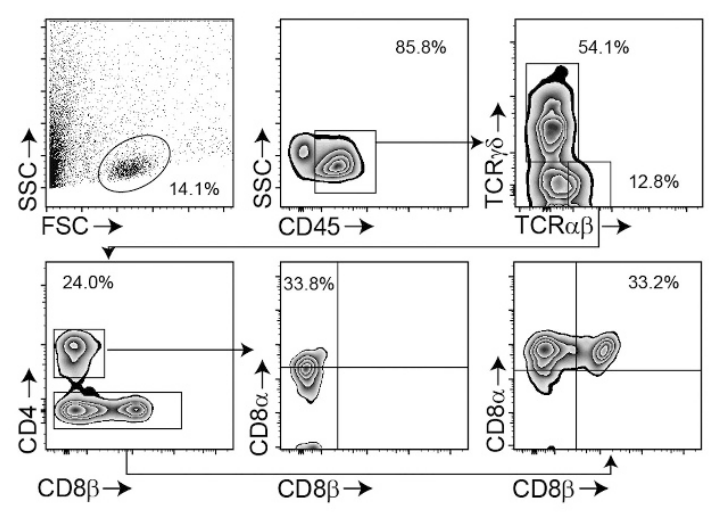

b

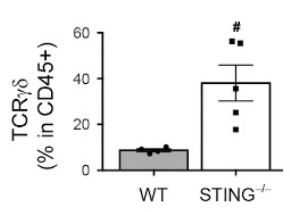

c
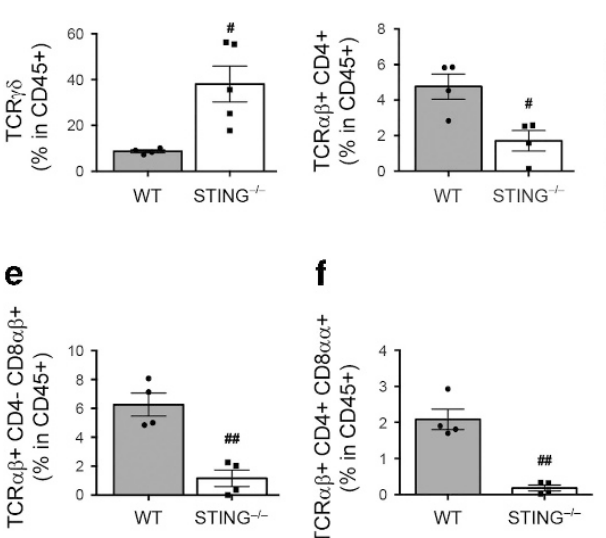

g

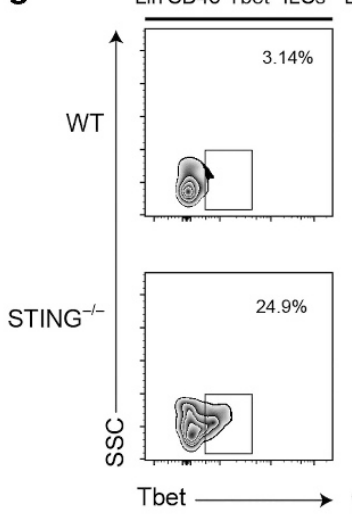

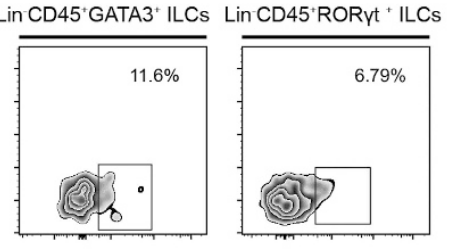
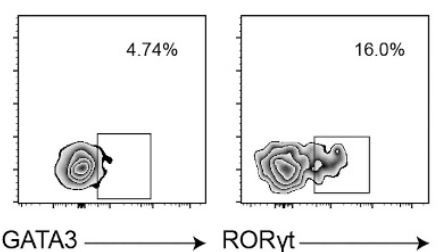
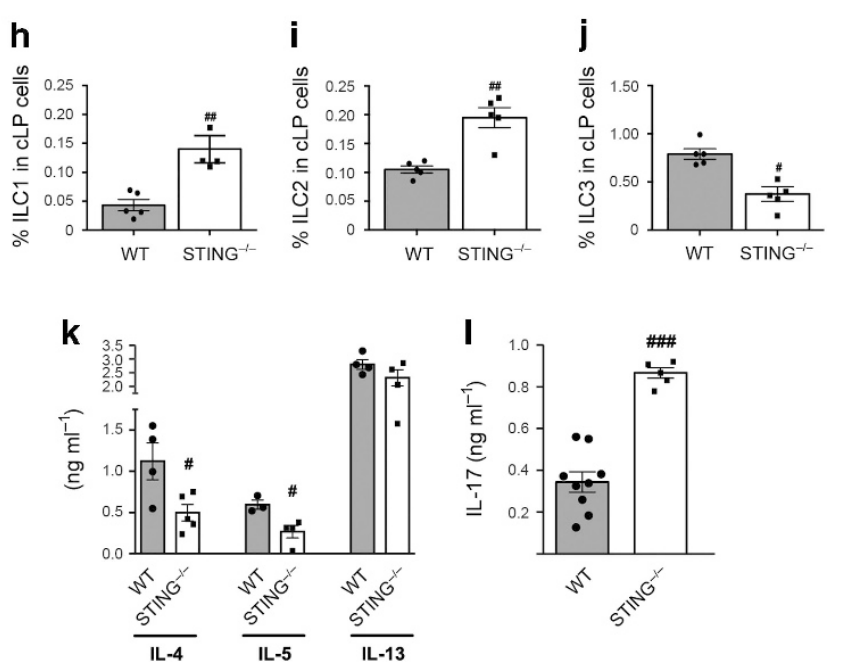

Figure 3 Altered frequency of intraepithelial lymphocytes (IELs) and innate lymphoid cells (ILCs) in STING ${ }^{-1-}$ mice. (a) Representative plots of IELs in the small intestine of wild-type (WT) and STING ${ }^{-1-}$ mice. Frequencies of (b) CD45 + TCR $\gamma \delta+$, (c) CD45 + TCR $\alpha \beta+$ CD $4+$, (d)

$\mathrm{CD} 45+\mathrm{TCR} \alpha \beta+\mathrm{CD} 4-\mathrm{CD} 8 \alpha \alpha+$, (e) CD $45+\mathrm{TCR} \alpha \beta+\mathrm{CD} 4-\mathrm{CD} 8 \alpha \beta+$, and (f) CD $45+\mathrm{TCR} \alpha \beta+\mathrm{CD} 4+\mathrm{CD} 8 \alpha \alpha+$ cells in the small intestine of WT and STING ${ }^{-1}$ mice. (g) Representative plots displaying frequencies of ILC1 (Lin-CD45 + CD127 + Tbet + ), ILC2 (Lin-CD45 + CD127 + GATA3 + ), and ILC3 (Lin-CD45 + CD127 + ROR $\gamma \mathrm{t}+$ ) in colon lamina propria of WT and STING ${ }^{-1}$ - mice. Frequencies of (h) ILC1, (i) ILC2, and (j) ILC3 cells in colon lamina propria of WT and STING ${ }^{-1-}$ mice. Levels of (k) interleukin (IL)-4, IL-5, and IL-13 and (I) IL-17 cytokines in colon extract of WT and STING ${ }^{-1-}$ mice. Data represent two independent experiments with four to five mice per group. Data represent the mean \pm s.e.m. ${ }^{\#} P<0.05 ;{ }^{\# \#} P<0.01 ;{ }^{\# \# \#} P<0.001$. FSC, forward scatter; SSC, side scatter.

frequency of $\mathrm{CD} 4+\mathrm{CD} 25+$ Foxp3 + and $\mathrm{CD} 4+\mathrm{LAP}+$ Treg cells in mesenteric lymph nodes (MLN) when compared with WT animals (Figure $\mathbf{4 b}-\mathbf{e}$ ). Consistent with that, levels of IL-10 and transforming growth factor (TGF)- $\beta$, critical for the expansion and maintenance of Foxp $3+$ and LAP + cells, respectively, were also reduced in the colon and MLN of STING $^{-1-}$ mice compared with WT (Figure $4 \mathbf{f}-\mathbf{i}$ ). These results suggest that STING signaling is required for the maintenance of a normal pool of Foxp3 + and LAP + Treg cells and optimal production of IL-10 and TGF- $\beta$ in MLN and LAP + Treg cells and TGF- $\beta$ in the colon.

Next, we examined whether STING signaling was also involved in Treg function by assessing their suppressive ability on T naive cells. Naive CD4 $+\mathrm{CD} 62 \mathrm{~L}+\mathrm{CD} 44^{\mathrm{low}}$ and CD4 + $\mathrm{CD} 25+$ Treg cells were sorted, co-cultured with antigenpresenting cells, and stimulated with anti-CD3 to induce T-cell proliferation. At the ratios of 1:1 and 1:2 of Treg:T naive cells, Tregs from WT mice were able to partially suppress $\mathrm{T}$-cell proliferation. However, Tregs from STING ${ }^{-1-}$ mice were unable to suppress proliferation of $\mathrm{T}$ cells at the same ratios (Figure 4j). Taken together, these data indicate that STING is essential for the maintenance and function of CD4 + Foxp3 + Treg cells.

\section{STING protected mice from different gut inflammatory insults}

Knowing that STING ${ }^{-1-}$ mice showed alterations in the gut that compromise the mechanisms of intestinal mucosa protection and immune-regulation, we assessed whether STING $^{-1-}$ mice presented increased susceptibility to intestinal inflammation. To address this question, we employed three different inflammatory insults: (a) a model of dextran sodium sulfate (DSS)-induced colitis in which intestinal inflammation is associated with epithelial destruction, self DNA release, and bacterial translocation; ${ }^{47}$ (b) a model of colitis induced by naive $\mathrm{CD} 4+\mathrm{CD} 45 \mathrm{RB}^{\text {high }}$ T-cell transfer into $\mathrm{RAG}^{-1-}$ mice in 
a
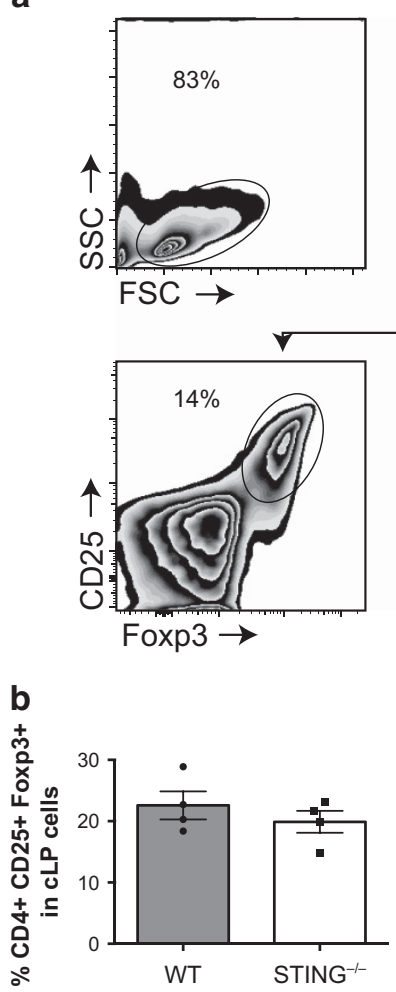

d

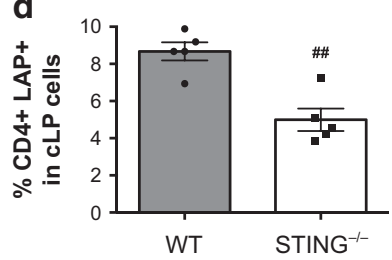

f

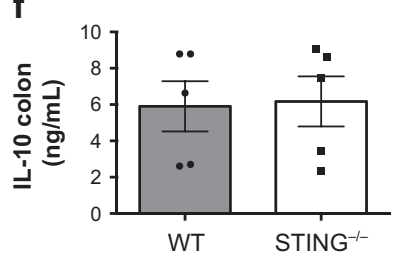

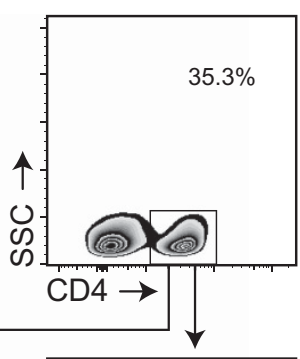

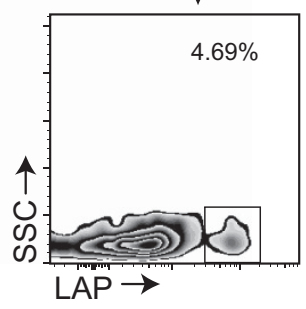

c

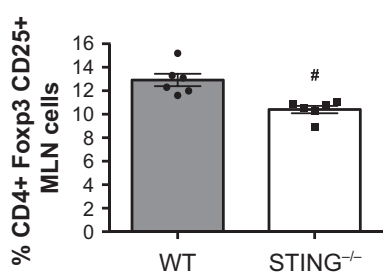

e

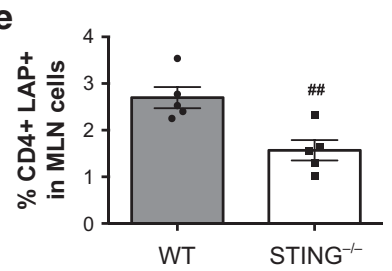

g

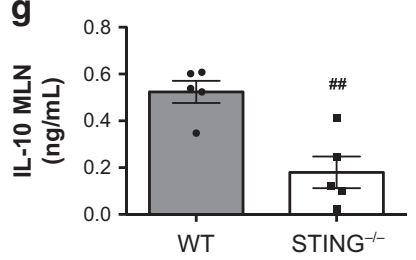

j
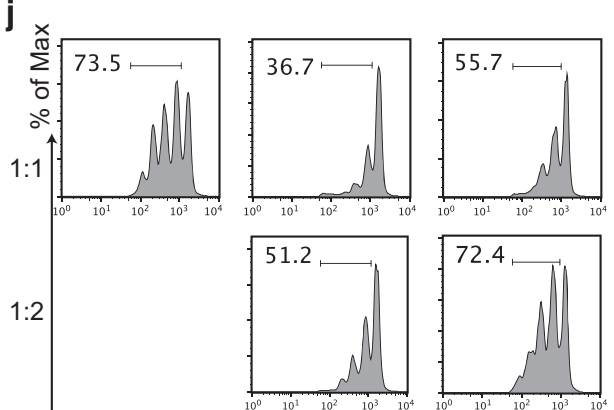

61.4
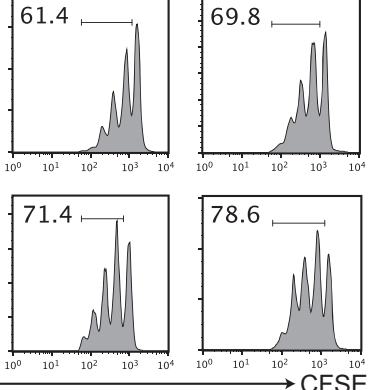

Q $\bullet A P C+$ Tnaive

\APC + Tnaive + WT Treg

$\square \cdot A P C+$ Tnaive + STING ${ }^{-1}$ Treg

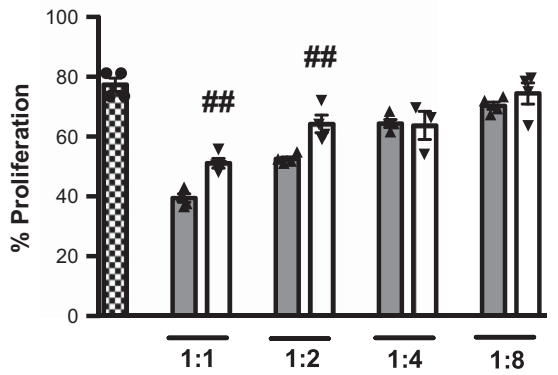

h

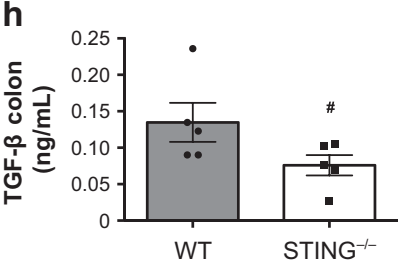

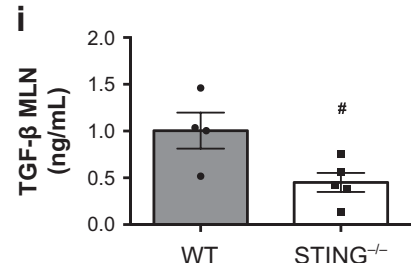

Figure 4 Frequency of regulatory T (Treg) cells in colon lamina propria (CLP) and mesenteric lymph nodes (MLN) of wild-type (WT) and STING ${ }^{-/-}$ mice. (a) Representative plots of gating strategy for Treg CD4 + CD25 + Foxp3 + and Treg CD4 + LAP + cells. (b) Frequency of Treg CD4 + CD25 + Foxp3 + in cLP and (c) MLN of WT and STING ${ }^{-\prime}$ mice. (d) Frequency of Treg CD4 + LAP + in cLP and (e) MLN of WT and STING ${ }^{-1-}$

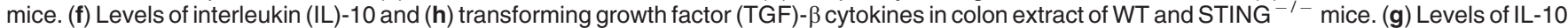
and (i) TGF- $\beta$ in MLN culture of cells stimulated with anti-CD3 and anti-CD28 for 48 and $72 \mathrm{~h}$, respectively. (j) CD4 + CD62L + CD44 ${ }^{\text {low }}$ or CD4 + CD25 + cells were purified by fluorescence-activated cell sorting from the spleen of 8-week WT and STING ${ }^{-/}$- mice and co-cultured with antigen-presenting cells (APCs) and anti-CD3 stimuli for 3 days at different proportions. Data represent two independent experiments with three to six mice per group. Data represent the mean \pm s.e.m. ${ }^{\#} P<0.05$; ${ }^{\# \# ~} P<0$.01. CFSE, carboxyfluorescein succinimidyl ester; FSC, forward scatter; LAP, latency-associated peptide; SSC, side scatter.

which co-transfer of functional regulatory $\mathrm{CD} 4+\mathrm{CD} 45 \mathrm{RB}{ }^{\text {low }}$ $\mathrm{T}$ cells prevents colitis development; ${ }^{48}$ and (c) a model of enteric infection by Salmonella thyphimurium.

WT and STING ${ }^{-1-}$ mice were treated with $3 \%$ DSS in drinking water for 8 days (followed by normal drinking water only in mortality experiments). Mice were monitored every day up to eighth or twenty-first day. STING ${ }^{-1-}$ mice showed increased weight loss (Figure 5a) bleeding, and diarrhea
(Figure 5b) starting on the fifth day when compared with WT mice. Moreover, all STING ${ }^{-1-}$ mice died within 13 days, whereas $30 \%$ of WT mice were still alive at day 13 and 8 days thereafter when the experiment was terminated (Figure 5c). Analysis of colon morphology and histology of the colons showed that their lengths were similar among control untreated animals, but DSS treatment led to a significant reduction in colon length, which was more evident in $\mathrm{STING}^{-1-}$ mice 


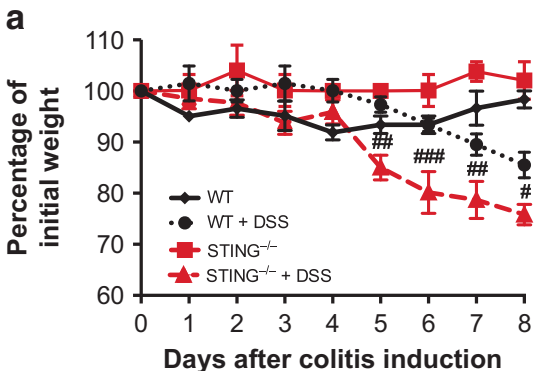

d

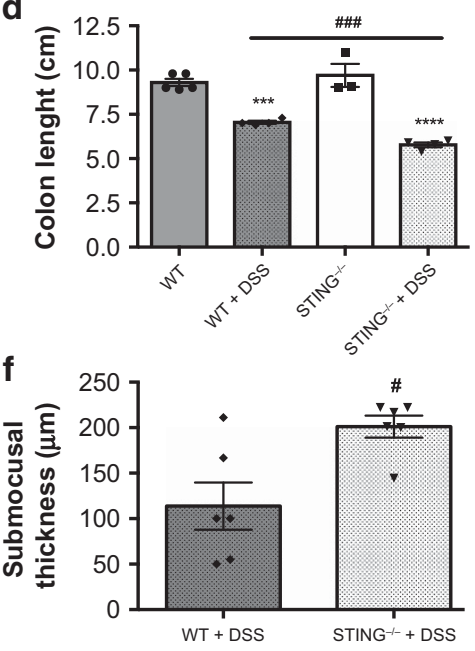

i

T cell-induced colitis

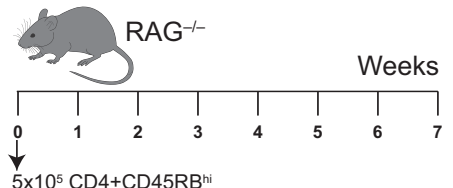

$5 \times 10^{5} \mathrm{CD} 4+C D 45 R B^{\text {hi }}+$ WT CD4+CD45RB low

$5 \times 10^{5} \mathrm{CD}+\mathrm{CD} 45 \mathrm{RB}^{\mathrm{hi}}+\mathrm{STING}^{-1-} \mathrm{CD} 4+\mathrm{CD} 45 \mathrm{RB}^{\text {low }}$

k

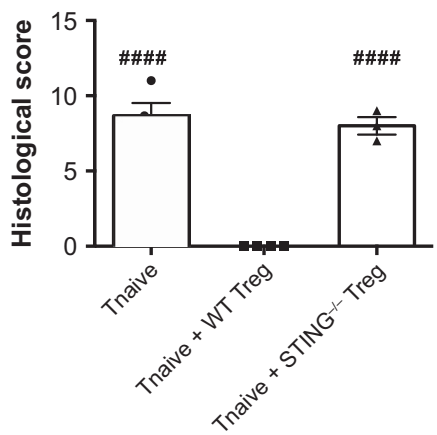

b

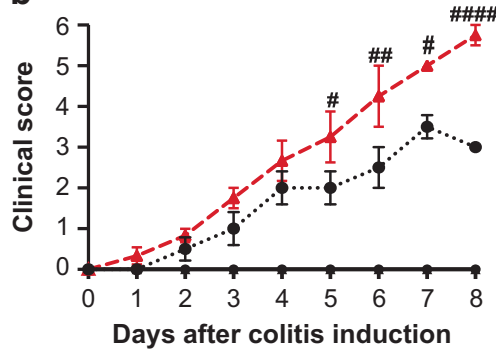

C

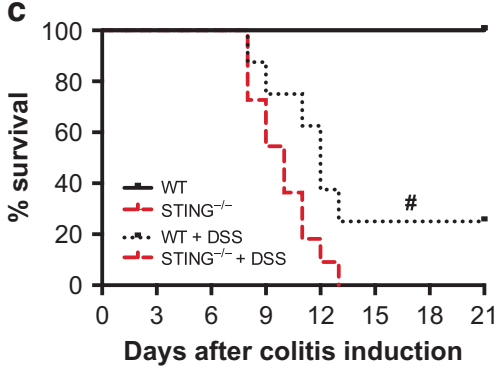

e

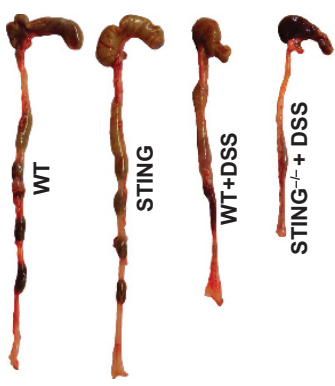

g
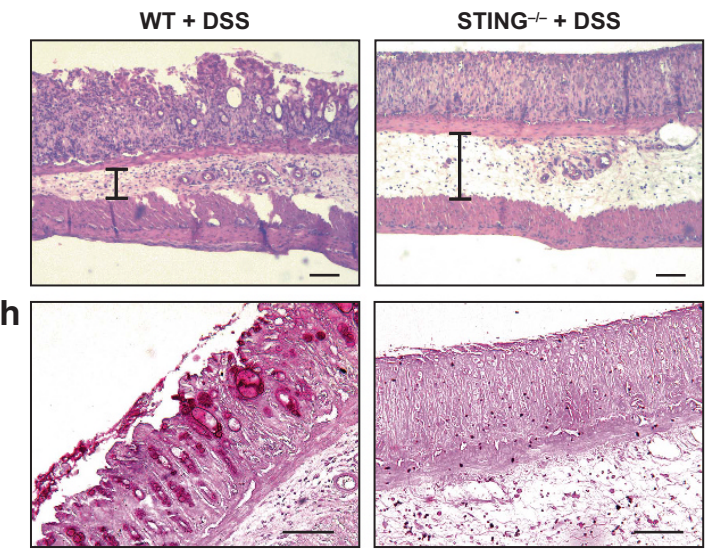

j
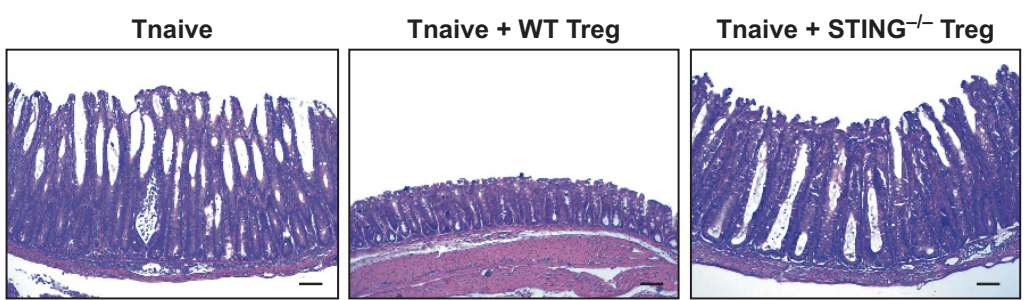

I

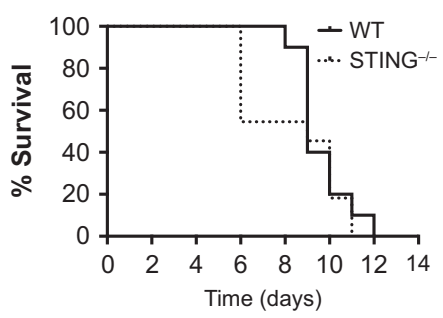

Figure 5 STING ${ }^{-1-}$ mice are highly susceptible to gut inflammation and enteric infection. Wild-type (WT) and STING ${ }^{-1-}$ mice were given $3 \%$ dextran sodium sulfate (DSS) in drinking water for 8 days. (a) Weight change following DSS administration, monitored every day. (b) Clinical score of WT and STING ${ }^{-1-}$ mice during DSS treatment, where a higher score corresponds to increased pathology. (c) Survival of WT and STING ${ }^{-1-}$ mice. Mice were given $3 \%$ DSS for 8 days, followed by normal drinking water for another 13 days, and monitored every day. (d) Quantification of the colon length described in e. (e) Colons from WT and STING ${ }^{-1-}$ mice on day 8. Representative photomicrographies of (g) hematoxylin and eosin (H\&E)-stained and (h) periodic acid-Schiff-stained colon sections from WT and STING ${ }^{-1}$ mice treated with DSS evidencing submucosal thickness and goblet cells. (i) Schematic protocol of colitis induced by transfer of naive CD4 $+\mathrm{CD}_{45 \mathrm{RB}^{\text {high }}} \mathrm{T}$ cells into RAG ${ }^{-1}$ - mice and adoptive co-transfer of CD4 + CD45RB ${ }^{\text {low }}$ Treg cells. (j) Representative photomicrographies of $\mathrm{H} \& \mathrm{E}$-stained colon sections from RAG ${ }^{-1}$ - mice that received WT T-naive cells (CD4 + CD45RB ${ }^{\text {high }}$ ) and cotransfer of either WT or STING ${ }^{-1}$ Treg cells (CD4 + CD45RB ${ }^{\text {low }}$ ). (k) Histological score of T-cell transfer colitis experiment represented in j. (I) WT and STING ${ }^{-1-}$ mice were treated with $1 \times 10^{8}$ colony-forming units of Salmonella typhimurium by gavage and their survival was assessed. Bars represent $100 \mu \mathrm{m}$. Data represent four independent experiments with 5-10 mice per group. Data represent the mean \pm s.e.m. ${ }^{\#} P<0.05$; ${ }^{\# \#} P<0.01$; $\# \#, * * * P<0.001 ; \# \# \#, * * * * P<0.0001$. 
(Figure 5d,e). Similar colitis clinical signs and colon length reduction was also observed in IFNAR-deficient mice (Figure 6a-f), suggesting that type I IFNs might participate in STING-dependent control of gut homeostasis. ${ }^{20,45,46}$ Histological hematoxylin and eosin staining of the colons showed that, after DSS treatment, STING ${ }^{-1-}$ mice had less intact epithelium and thicker submucosal layer than the WT mice (Figure 5f,g) indicating more edema. Periodic acid-Schiff staining also showed remaining goblet cells and intestinal architecture in WT mice compared with STING ${ }^{-1-}$ after DSS treatment (Figure 5h). Moreover, we observed lower frequencies of $\mathrm{CD} 4+\mathrm{CD} 25+$ Foxp $3+$ and $\mathrm{CD} 4+\mathrm{LAP}+$ Treg cells in colonic lamina propria and MLN of STING ${ }^{-1-}$ mice (see Supplementary Figure S4a,b,e,f) and reduced levels of TGF- $\beta$ (see Supplementary Figure $\mathbf{S 4 c , d , g , h}$ ) when compared with WT animals after DSS treatment.

STING expression by Treg cells was necessary for their suppressive function in vitro (Figure 4j). Therefore, we used a model of gut inflammation in which functional Treg cells are necessary to prevent the development of T-cell-induced colitis $^{48}$ to test Treg function in vivo. $\mathrm{RAG}^{-1-}$ mice transferred with naive $\mathrm{CD} 4+\mathrm{CD} 45 \mathrm{RB}^{\text {hi }} \mathrm{T}$ cells showed histological alterations that were compatible with inflammation in gut mucosa as shown by histological analysis (Figure 5i-k), whereas co-transfer of WT CD4 + CD45RB ${ }^{\text {low }}$ Treg cells with naive $\mathrm{CD} 4+\mathrm{CD} 45 \mathrm{RB}^{\text {high }} \mathrm{T}$ cells prevented development of disease. However, co-transfer of STING $^{-1-}$ $\mathrm{CD} 4+\mathrm{CD} 45 \mathrm{RB}^{\text {low }}$ Treg cells failed to prevent disease development, confirming the in vitro results that STING expression on Treg cells is required to its suppression function.

To investigate mouse susceptibility to enteric pathogen infection in the absence of STING, we examined the mortality rate of WT and STING ${ }^{-1-}$ mice following the oral administration of $1 \times 10^{8}$ colony-forming units of Salmonella typhimurium. STING ${ }^{-1-}$ mice displayed early mortality rate compared with WT mice (Figure 5l). On day 6 after infection, $60 \%$ of STING ${ }^{-1-}$ mice had died while WT mice start to die only from day 9 after infection. We also analyzed bacteria translocation at day 6 after infection and observed similar bacterial loads in the liver of WT and STING ${ }^{-1-}$-infected mice (data not shown), indicating that even though bacterial translocation is not different between both groups, STING $^{-1-}$ mice are more susceptible to Salmonella infection.

Taken together, these results showed that STING is a critical regulator of intestinal homeostasis and that STING-deficiency leads to higher susceptibility to gut inflammation and to enteric infection in mice.

\section{DISCUSSION}

A variety of pattern-recognition receptors expressed in intestinal epithelial cells and immune cells maintain the surveillance of pathogen-associated molecular patterns and trigger homeostatic immune responses allowing the successful coexistence of the gut commensal microbiota with an intact intestinal mucosal tissue. Microbial DNA is known to be sensed by TLR9, AIM2, and cGAS, leading to the activation of NF- $\mathrm{KB}$, inflammasome, and IFN signaling pathways, respectively. ${ }^{7,13,49}$ TLR9- and AIM2-mediated recognition of DNA in the intestine exerts a protective effect against experimental colitis. ${ }^{7,49,50}$ Although the physiological relevance of DNA sensing by cGAS during intestinal inflammation is still unclear, type I IFN signaling has been shown to be critical in regulating epithelial and T-cell functions. ${ }^{20,45,46,51}$ However, little is known about the role of STING as a sensing of CDNs and as an adaptor for DNA sensing in intestinal homeostasis.

Intestinal mucosa harbors fast renewing epithelial cells that are in contact with external stimuli, such as microbiota. ${ }^{6,7}$ Both components, dying cells and bacteria from microbiota, are continuous sources of free DNA that can deliver physiological stimulation to immune cells. Studies on germ-free mice showed that these animals present several signs of immunological and intestinal immaturity when compared with conventional mice, including elongated and smooth villi and smaller colon crypts, as well as reduced production of mucus and SIgA with normal levels of serum IgM. ${ }^{28,31,52}$ The role of mucus layer and SIgA in gut homeostasis is clear. The intestinal mucus layer provides a barrier limiting bacterial contact with the underlying epithelium and SIgA reduces the penetration of commensal bacteria by preventing their adhesion to the epithelium. ${ }^{53}$ Thus the presence of a normal intestinal microbiota positively influences immune responses and protects against the development of inflammatory diseases by inducing the development of several intestinal and immune-protective mechanisms since early life. Indeed, it has been shown that germ-free mice are highly susceptible to DSS-induced colitis. ${ }^{31}$ On the other hand, it has also been described that products of immune cells such as SIgA in the gut can influence bacterial composition of the local microbiota. ${ }^{34,35}$ These reports suggest that there is a circuit of mutual regulation between immune cells and the microorganisms that colonize the gut mucosa. Analysis of microbiota composition in STING ${ }^{-1-}$ mice show that the absence of this signaling pathway does not change the ratio of Firmicutes to Bacteroidetes phyla. However, alterations in specific genera of bacteria resulted in a significant change in microbiome composition and in a profile related to susceptibility to gut inflammation. ${ }^{54}$ A divergent shift was observed within the phylum Firmicutes, with a decrease in Allobaculum in feces from STING ${ }^{-1-}$, a group of bacteria that is induced by type I IFN and is related with reduction of IL-17 production. ${ }^{38}$ STING $^{-1-}$ mice had also an increase in Desulfovibrio genus (Proteobacteria phylum), a Gram-negative bacterium whose prevalence have been shown to be associated with ulcerative colitis. ${ }^{36}$ On the other hand, a reduction in Bifidobacterium (Actinobacteria phylum), a species with anti-inflammatory effects mediated by TGF- $\beta$ and Treg cells in the gut, ${ }^{55}$ was also observed.

Although STING ${ }^{-1-}$ mice bear a microbiota, they miss a critical signaling pathway for microbiota DNA and CDNs. Many species of gut bacteria produce cyclic dinucleotides ${ }^{26}$ and therefore may stimulate STING. Indeed, our results show that STING $^{-1-}$ mice present a phenotype that resembles the one observed in germ-free mice. They had longer and less crimped 


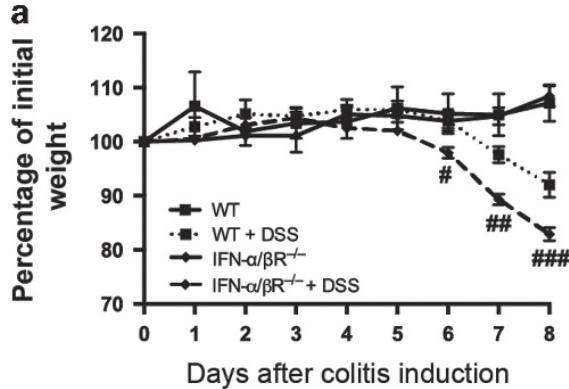

d

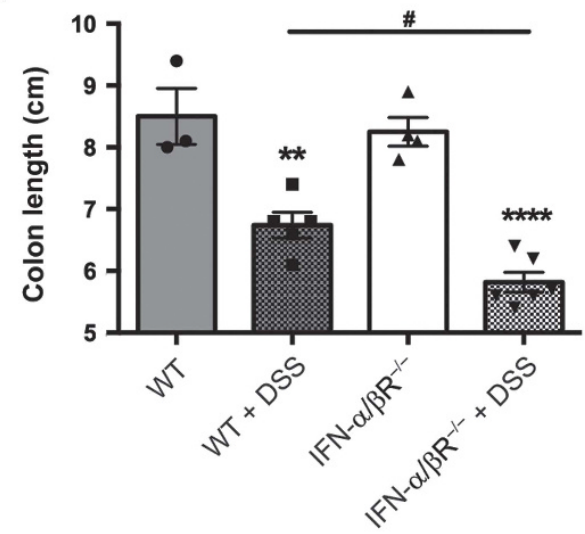

b

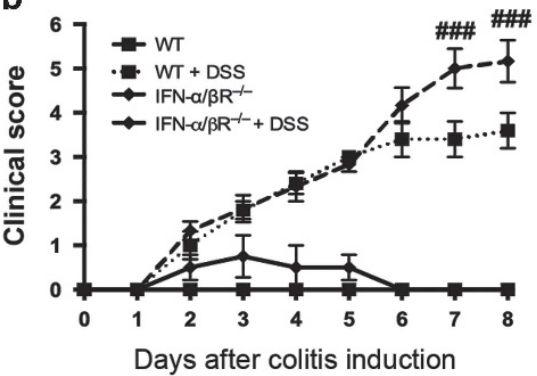

e

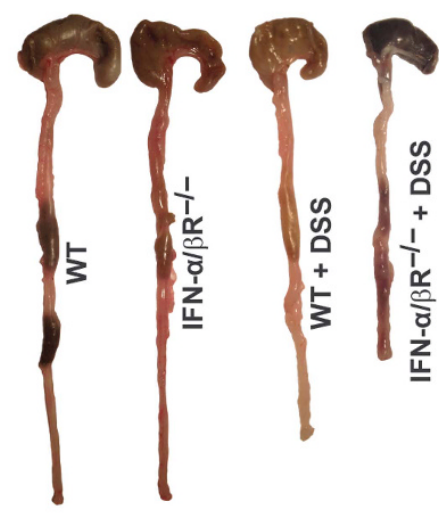

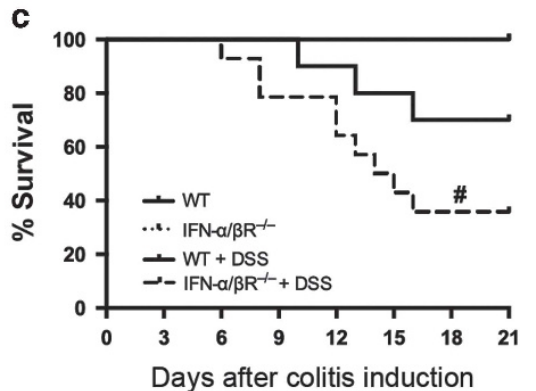

f

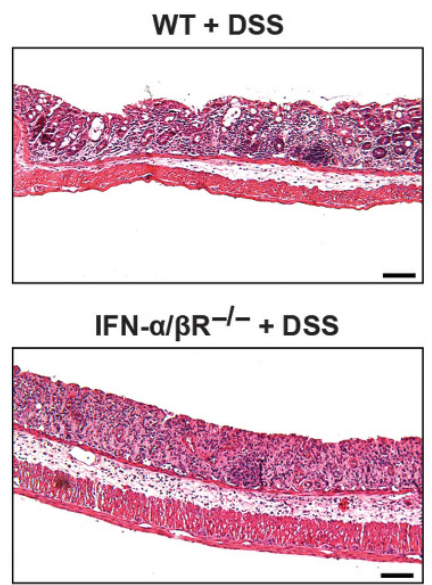

Figure 6 Interferon (IFN)- $\alpha / \beta R^{-1-}$ mice are also susceptible to dextran sodium sulfate (DSS)-induced colitis. (a) Weight change following DSS administration, monitored everyday. (b) Clinical score of wild-type (WT) and IFN- $\alpha / \beta R^{-1-}$ mice during DSS treatment, where a higher score corresponds to increased pathology. (c) Survival of WT and IFN- $\alpha / \beta R^{-1-}$ mice. Mice were given $3 \%$ DSS for 8 days, followed by normal drinking water for another 13 days, and monitored everyday. (d) Quantification of the colon length described in e. (e) Colons from WT and IFN- $\alpha / \beta R^{-1-}$ mice on day 8. Data are representative of 3-10

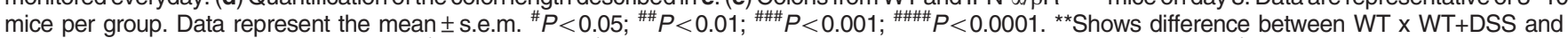

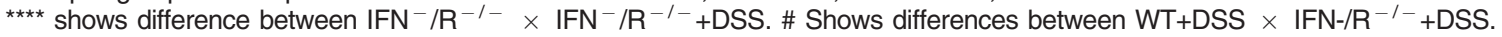

small intestine villi, smaller colon crypts, lower production of MUC1/MUC2, lower levels of SIgA, and increased serum IgM levels (data not shown) when compared with WT mice. The immaturity of gut mucosa is also consistent with the profile of IELs in STING ${ }^{-1-}$ mice. As already described in neonates and germ-free mice, ${ }^{40}$ STING $^{-1-}$ mice presented a decreased frequency of all subsets of induced IELs (expressing TCR $\alpha \beta$ ). IELs are T lymphocytes interspersed in the gut epithelium as a first line of immune protection against pathogens. They are present as distinct subpopulations depending on the T-cell receptor (TCR) rearrangement they show $(\alpha \beta$ vs. $\gamma \delta)$ and on the expression of either homodimeric $(\alpha \alpha)$ or heterodimeric $(\alpha \beta)$ form of CD8 as well as co-expression of CD4. ${ }^{56}$ Regardless of CD8 and CD4 expression, we found that all subsets of induced TCR $\alpha \beta$-expressing IELs are downmodulated and natural TCR $\gamma \delta$-expressing IELs are increased in frequency in the absence of STING signaling. This result is in concert with the role of microbiota in inducing TCR $\alpha \beta+$ IELs and also with their role in protection against infection. ${ }^{40}$ Indeed, STING ${ }^{-1-}$ mice were more susceptible to Salmonella infection than WT mice although intestinal permeability and bacteria translocation (data not shown) were not changed in STING ${ }^{-1-}$ mice. It is likely that the compromised mucosal protective immunity is a net result of defects in several components of barrier function in these animals.
The pattern of ILCs in neonates and germ-free animals is less explored. Recently, these innate lymphocyte lineages, previously thought to be represented only by natural killer cells, were described and are now referred to as ILCs. ILCs are preferentially located at barrier surfaces and are important for protection against pathogens and for the maintenance of organ homeostasis. Inappropriate activation of ILCs has been linked to the pathogenesis of inflammatory and autoimmune disorders. ${ }^{41}$ As fetal lymphoid inducer cells and lymphoid inducer-like cells are part of the ILC3 population, we would expect to have an overrepresentation of this subset at early stages of development. These cells have been shown to be the first immune cells to seed the intestinal mucosa. ${ }^{57}$ In concert with our hypothesis that STING $^{-1-}$ present an immature immunological profile, the frequency of ILC3 is increased in STING ${ }^{-1-}$ mice when compared with WT mice. Moreover, analysis of frequencies of ILCs in the intestinal mucosa of STING ${ }^{-1-}$ and WT neonates showed no difference between the two groups. It is plausible that microbiota colonization has a critical role in delivering stimulatory cues such as cyclic DNA for the development of the adult profile of ILCs seen in WT mice. On the other hand, STING $^{-l-}$ mice presented a lower frequency of ILC2 when compared with age-matched WT mice. ILC2 was shown to mediate tissue protection during intestinal injury, such as DSS-induced colitis, by limiting inflammation and promoting 
epithelial repair through amphiregulin production. ${ }^{58}$ Differentiation of these cells requires IL-25, IL-33, and thymic stromal lymphopoietin (TSLP). IL-33 and Tslp induction is markedly decreased in Sting knockdown cells. ${ }^{59}$ Moreover, it was shown that IRF3, which is activated by STING, is involved in IL-33 and TSLP production and IRF3 ${ }^{-l-}$ mice are also more susceptible to DSS-induced colitis. ${ }^{59} \mathrm{We}$ observed that the colonic lamina propria of STING ${ }^{-1-}$ mice had a decreased frequency of ILC2, reduced levels of Amphiregulin mRNA, and increased frequency of ILC1 and ILC3. Although ILC3 can be protective in some types of intestinal disorders, detrimental ILC1 and ILC3 responses have been reported in models of colitis induced by Helicobacter hepaticus or anti-CD40 treatment and have been related to IL-22, IL-17, granulocyte macrophages colonystimulating factor, and/or IFN- $\gamma$ activity. ${ }^{60,61}$ Moreover, increased frequency of ILC1 is observed in patients with Crohn's disease. ${ }^{62}$ Our findings suggest that increased frequency of ILC1 and ILC3 seems to cooperate with the decreased ILC2 in colonic lamina propria of STING ${ }^{-1-}$ mice to enhance their susceptibility to colitis. Thus a balance in ILC subtype frequency in the colon is essential to maintain gut homeostasis.

Along with these innate and acquired immune mechanisms of barrier maintenance, the intestinal mucosa lodges distinct subsets of Treg cells that are essential for the induction of specific immunological tolerance to gut bacteria. Their absence or failure to patrol the intestinal mucosa leads to reactivity against the commensal microbiota and development of inflammatory bowel disease in mice and humans. ${ }^{63,64} \mathrm{An}$ important regulator of CD4 + Foxp3 + Treg cells is the type I IFNs. The absence of IFNAR signaling on innate immune cells result in poor expansion and maintenance of Foxp $3+$ Tregs in the intestine, leading to the outgrowth of Th1 and Th17 effector cells in the inflamed colon. ${ }^{20}$ STING pathway is known to induce the production of type I IFNs, and we confirmed that showing that STING ${ }^{-1-}$ mice produce significantly lower levels of IFN- $\beta$ mRNA transcripts in the colon than WT mice. Consistent with the effects of type I IFNs in Treg expansion and maintenance, we observed that the frequency of $\mathrm{CD} 4+$ CD25 + Foxp3 + was reduced in MLN and the frequency of TGF- $\beta$-producing CD $4+\mathrm{LAP}+$ Tregs was reduced in the colonic lamina propria and MLN of STING ${ }^{-1-}$ mice when compared with their WT counterparts. Moreover, absence of STING signaling also interfered with the function of CD4+ CD25 + Foxp 3 - Treg cells, as they were less efficient in suppressing T-cell proliferation in vitro and in preventing colitis induction when co-transfered with naive CD4 $+\mathrm{T}$ cells into $\mathrm{RAG}^{-1-}$ mice. $^{48} \mathrm{We}$ speculate that the alterations observed in STING ${ }^{-1-}$ Treg cells were due to the lower levels of type I IFN in those animals; however, we cannot discard the possibility that STING is interfering in Treg development and function in a type I IFN-independent manner. On the other hand, the critical role of IFN- $\beta$ in the maintenance of gut homeostasis and protection against inflammation was underscored in the present study by the increased susceptibility of both STING ${ }^{-1-}$ mice and IFN $\alpha / \beta$ $\mathrm{R}^{-1-}$ to colitis induction by DSS.
Interestingly, regulatory cells and cytokines were differentially altered in MLN and colonic lamina propria of STING ${ }^{-1-}$ mice. TGF- $\beta$ and CD $4+\mathrm{LAP}+$ cells were both reduced in the colon, whereas CD4 + CD25 + Foxp3 + Tregs, CD4 + LAP + cells, and IL-10 production were all reduced in MLN. MLN drain the lamina propria of the small intestine but not the colonic lamina propria. Several immune-regulatory factors are probably activated at distinct sites of the intestine and they may vary according to the specific features and requirements of the region ${ }^{65}$ as well as additional factors. ${ }^{66}$ It is also plausible that other signaling molecules for relevant microbiota stimuli, including DNA (such as TLR9, AIM2, and so on) are also differentially expressed along the gut mucosa having complementary roles in conveying cues from the microbiome for the proper differentiation of regulatory cells, such as IL-10producing cells.

STING signaling can also activate NF- $\kappa \mathrm{B}$, which induces the transcription of many genes, including mucins. NF- $\mathrm{KB}$ induces the production of MUC2 by human colonic epithelial cells $s^{67,68}$ and by human epithelial cell lines in cooperation with TGF- $\beta-S m a d$ signaling pathway. ${ }^{69}$ Although we did not assess NF- $\mathrm{KB}$ activation here, it is well described that STING signaling pathway induces NF- $\kappa B$ translocation to the cell nucleus. ${ }^{11,14,16}$ Moreover, we observed lower levels of TGF- $\beta$ in the colon of STING ${ }^{-1-}$ mice, which suggests that the reduced MUC2 production observed in these animals could be due to an additive effect of poor activation of NF- $\kappa B$ and low levels of TGF- $\beta$. MUC2 is also able to induce the production of IL-10 and TGF- $\beta$ by tolerogenic DCs involved in the induction of Treg cells. ${ }^{70}$ Thus a dense mucus layer in the large intestine prevents inflammation not only by shielding the underlying epithelium from luminal bacteria and food antigens but also by delivering tolerogenic signals. Studies showing that $\mathrm{MUC2}^{-1-}$ mice develop spontaneous colon inflammation underscore the critical role of MUC2 in gut homeostasis. ${ }^{71}$

STING and CDNs are highly conserved throughout evolution, being observed $>500$ million years ago in the common ancestor of human and Cnidaria. ${ }^{72}$ CDNs are secondmessenger signaling molecules produced by diverse bacterial species, including gut commensal microbiota, ${ }^{25,73}$ and are also synthesized by cytosolic cGAS upon dsDNA binding. The modern features of innate immunity appeared later in evolution than the STING-CDN binding, suggesting that STING-CDN pathway may have a function other than its role in host defense against pathogens. We hypothesized that the microbiota CDNs sensed by STING might act by inducing a physiological tonus of activation for protective mechanisms involved in intestinal homeostasis. It is possible that the 3'3" $\mathrm{CDNs}$ evolved to be a less potent STING agonist in mammals in order to keep gut homeostasis without initiating an immune response against the microbiota. During pathogen infection, however, STING is strongly activated by 2', 3' cGAMP produced by cGAS, a more potent STING agonist, ${ }^{74}$ and a proper inflammatory immune response against pathogen is triggered. This observation merits further investigation. 
The cytosolic adaptor STING has important function in the host defense against pathogens and in autoimmune diseases; however, its role in gut homeostasis has been largely unknown. This study reveals that STING can be activated by DNA present in fecal contents, and this adaptor molecule has a critical function in the development of gut-protective mechanisms, such as mucus and SIgA production, in the balance of ILC subsets and in the Treg cell activity in the intestinal mucosa. As a consequence, STING ${ }^{-1-}$ mice had a higher susceptibility to DSS-induced and T-cell-induced colitis as well as to enteric infection. Therefore, our data show that STING is an important signaling molecule involved in gut homeostasis and may also serve as a therapeutic target for the regulation of intestinal inflammation.

\section{METHODS}

Mice. STING ${ }^{-1-}$ mice were obtained from Dr Glen Barber (University of Miami, Miami, FL) and maintained in the animal care facility of Universidade Federal de Minas Gerais (UFMG). C57BL/6 WT, 129 Sv/Ev WT, STING ${ }^{-1-}, \mathrm{IFN}_{-} \alpha / \beta \mathrm{R}^{-1-}$, and $\mathrm{RAG}^{-1-}$ mice were bred and maintained in the animal care facility of the Laboratório de Imunobiologia at UFMG as previously described. ${ }^{41}$ Male and female mice used in experiments were aged 7-12 weeks. All conventional mice within an experiment were cohoused (gender matched) for 4 weeks to minimize differences among their microbiotas. Germ-free mice were obtained from the Gnotobiotic facility of UFMG, and they were maintained there in isolators for the entire period of the experiment. All mice were housed and used according to the experimental protocols approved by the Ethics Committee on Animal Care and Use (CEUA) of UFMG.

Induction of colitis with DSS. Male or female C57BL/6 WT and $\mathrm{STING}^{-1-}$ mice or $129 \mathrm{~Sv} / \mathrm{Ev} \mathrm{WT}$ and IFN- $\alpha / \beta \mathrm{R}^{-1-}$ mice were cohoused for 4 weeks before colitis induction. Animals received 3\% DSS (molecular weight 36,000-50,000; MP Biomedicals, Santa Ana, CA) in drinking water for 8 days. There was no difference in the amount of DSS consumption between experimental groups. For survival studies, mice were monitored every day up to 21 days postinitiation of DSS treatment. For weight-change studies, the percentage of initial weight was determined through the following equation: percentage of initial weight: (weight at the $x$ th day $\times 100$ )/ (weight on day 0). Animals were also monitored for feces consistency, rectal bleeding, diarrhea, and general signs of morbidity, including hunched posture and failure to groom, and scored between 0 and 6 , with 0 being normal up to 6 being diarrhea, signs of morbidity, and failure to groom.

Flow cytometric analysis. Cells were isolated from colon lamina propria by removing the entire length of colon, dissected, opened longitudinally, washed with phosphate-buffered saline (PBS), and cut into small pieces. Tissue fragments were placed in Petri dishes and washed in calcium and magnesium-free Hank's Balanced Salt Solution containing $2 \%$ fetal bovine serum. Then fragments of colon lamina propria were transferred to culture flasks and incubated at $37^{\circ} \mathrm{C}$ in Hank's Balanced Salt Solution containing $1 \mathrm{~mm}$ DL-dithiothreitol (Sigma, St Louis, MO) for $60 \mathrm{~min}$. After that, tissue fragments were then incubated with $100 \mathrm{U} \mathrm{ml}^{-1}$ of collagenase II (Sigma) for $60 \mathrm{~min}$ at $37^{\circ} \mathrm{C}$ in a shaker. Supernatants were passed through a $70-\mu \mathrm{m}$ cell strainer and ressuspended in medium. Lamina propria cells were then preincubated with anti-CD16/CD32 monoclonal antibody to block Fc $\gamma$ RII/III receptors and stained on ice for $30 \mathrm{~min}$ with the following fluorochrome-conjugated monoclonal antibody: fluorescein isothiocyanate-labeled anti-CD4, PE-labeled anti-LAP, PerCPCy5.5labeled anti-CD25, and APC-labeled anti-Foxp3 for Treg cells analysis.
Pacific Blue-labeled anti-CD11b, anti-CD11c and anti-FceR1 $\alpha$; BV421-labeled anti-CD16/32 and anti-CD19; PerCPCy5.5-labeled anti-CD8; PeCy7-labeled anti-CD4; BV570-labeled anti-CD45; BV510-labeled antiCD127; Aexa700-labeled anti-CD3; PE-CF594labeled anti-ROR $\gamma \mathrm{t}$; PE-labeled anti-T-bet, and AlexaFluor 488labeled anti-GATA3 were used for T-cell and ILC analysis. Antibodies were purchased from Invitrogen, Carlsbad, CA; BD Pharmingen (San Jose, CA); or R\&D Systems, Minneapolis, MN. Surface staining was performed according to standard procedures at a density of $1 \times 10^{6}$ cells per $25 \mu \mathrm{l}$, and volumes were scaled up accordingly. Flow cytometric analysis was performed on a FACSCanto (BD Biosciences, San Jose, CA) or on a FACSFortessa (BD Biosciences).

Cell sorting. A pool of cells from spleen of WT and STING ${ }^{-/-}$mice was first enriched using CD4 + Microbeads T-Cell Isolation Kit (Miltenyi Biotec, Bergisch Gladbach, Germany). Naive CD4 + CD62L + CD $44^{\text {low }}$ $\mathrm{T}$ cells and $\mathrm{CD} 4+\mathrm{CD} 25+$ Treg cells were sorted (FACSAriaII, BD Biosciences) with PeCy-7-labeled anti-CD4, APC-labeled anti-CD62L, PE-labeled anti-CD44, and PerCPCy5.5-labeled anti-CD25.

Treg suppression assay in vitro. Sorted Treg CD4 + CD25 + cells were cultured at $1: 1,1: 2,1: 4$, and 1:8 ratio with $\mathrm{T}$ naive CD4+ CD62L + CD $44^{\text {low }}$ cells previously stained with carboxyfluorescein succinimidyl ester dye (Invitrogen). Cells were stimulated with antiCD3e $\left(1 \mu \mathrm{g} \mathrm{ml}^{-1}\right.$; 145-2C11, BioLegend, San Diego, CA) in the presence of antigen-presenting cells in $200 \mathrm{ml}$ of RPMI medium supplemented with $10 \%$ fetal bovine serum in 96-well round-bottom plates. Proliferation and Foxp3 induction were assessed $72 \mathrm{~h}$ later by flow cytometry (FACSCalibur, BD Biosciences), based on the dilution of the carboxyfluorescein succinimidyl ester dye.

Cell culture preparation. MLN were removed and cells suspensions were prepared using a tissue homogenizer, being gently centrifuged. Cells isolated from MLN were cultured, at $1 \times 10^{6}$ cells per well for cytokine secretion analyses, in 98-well plates in complete RPMI and stimulated or not with $1 \mu \mathrm{g} \mathrm{ml}^{-1}$ of anti-CD3 and anti-CD28. Supernatants were collected after $48 \mathrm{~h}$ to measure IL-10 and after $72 \mathrm{~h}$ to measure TGF- $\beta$.

T-cell transfer model of colitis. Naive CD $4+C D 45 R B$ hi $T$ cells were isolated from WT or STING ${ }^{-1-}$ mice and injected intraperitoneally into 7-8-week-old $\mathrm{RAG}^{-1-}$ immune-deficient recipients $\left(5 \times 10^{5}\right.$ cells per mouse). WT and STING ${ }^{-1-}$ CD $4+\mathrm{CD}_{4} 4 \mathrm{RB}^{\text {low }}$ Treg cells $\left(2.5 \times 10^{5}\right.$ mouse $)$ were co-injected intraperitoneally as previously described. ${ }^{75}$ Mice were monitored weekly for wasting disease, and any mice losing $>20 \%$ of its starting body weight or showing severe signs of disease were killed.

Quantitative reverse transcriptase-PCR. Colon tissue or cultured lamina propria cells were homogenized in TRIzol (Invitrogen), and RNA was isolated by phenol chloroform extraction and isopropanol precipitation. Reverse transcription of $2 \mu \mathrm{g}$ of total RNA was performed using Illustra Ready-To-Go reverse transcriptase-PCR (RTPCR) Beads (GE Healthcare, Little Chalfont, UK) according to the manufacturer's instructions. Real-time RT-PCR was conducted with a final volume of $10 \mu \mathrm{l}$ containing SYBR green PCR Master Mix (Applied Biosystems, Carlsbad, CA), oligo(dT) cDNA as the PCR template and $10 \mu \mathrm{M}$ primers. Real-time RT-PCR was performed with the ABI7900 real-time PCR system (Applied Biosystems, Foster City, CA) using the following cycling parameters: $60^{\circ} \mathrm{C}$ for $10 \mathrm{~min}, 95^{\circ} \mathrm{C}$ for $10 \mathrm{~min}, 45$ cycles of $95^{\circ} \mathrm{C}$ for $15 \mathrm{~s}$, and $60^{\circ} \mathrm{C}$ for $1 \mathrm{~min}$, and a dissociation stage of $95^{\circ} \mathrm{C}$ for $15 \mathrm{~s}, 60^{\circ} \mathrm{C}$ for $1 \mathrm{~min}, 95^{\circ} \mathrm{C}$ for $15 \mathrm{~s}$, and $60^{\circ} \mathrm{C}$ for $15 \mathrm{~s}$. The primers used for the $\beta$-actin, IFN- $\beta$, and amphiregulin genes were as follows: $\beta$-actin F: $5^{\prime}$-GGCTGTATTCCCCTCCATCG-3' and R:5"CCAGTTGGTAACAATGCCATGT- $3^{\prime} ; \quad$ IFN- $\beta$ F:5'-GCCTTTGC CATCCAAGAGATGC- $3^{\prime}$ and R: $5^{\prime}$-ACACTGTCTGCTGGTGGAG TTC-3' . Amphiregulin F: 5'-GCCATTATGCAGCTGCTTTGGAGC$3^{\prime}$ and R: $5^{\prime}$-TGTTTTTCTTGGGCTTAATCACCT- $3^{\prime}$. All data are presented as relative expression units after normalization to $\beta$-actin. 
Histology and immunohistochemistry. Animals were killed and their colons were collected and opened longitudinally. For colitis analysis, on day 8 after 3\% DSS treatment or 7 weeks after T-cell transfer to $\mathrm{RAG}^{-1-}$ mice, the colon of mice were also extracted. Later the colons were fixed with $10 \%$ formalin in neutral buffer and embedded in paraffin. Histological sections were deparaffinized and stained with hematoxylin and eosin or Periodic acid-Schiff and analyzed by light microscopy. For immunohistochemical analysis, consecutive $4-\mu \mathrm{m}$-thick sections were mounted on silanized slides, and peroxidasebased detection system Advance HRP was applied (Dako North America, Carpinteria, CA). Antigen retrieval was performed in water bath at $98^{\circ} \mathrm{C}$, with a citrate buffer solution ( $\mathrm{pH}$ 6.0) (Target Retrieval Solution) for $20 \mathrm{~min}$. Endogenous peroxidase activity was blocked with a $3 \%$ hydrogen peroxide solution in methyl alcohol. Sections were stained with anti-MUC1 (1:50; Abcam, Cambridge, UK) and antiMUC2 (1:300; Abcam), and immunoreactivity was visualized by incubating the slides with diaminobenzidine (DAB substrate system, Lab Vision, Fremont CA) for $10 \mathrm{~min}$. The histological changes were analyzed in a double-blinded manner. Immunohistochemical analysis of MUC2 were based on scores divided into frequency of the positive cells in 10 fields $(60 \times)$, classified as: $1+$ when $0-25 \%$ of cells staining; $2+$ when $25-50 \%$ of cells staining; $3+$ when $50-75 \%$ of cell staining; and $4+$ when $>75 \%$ of cells staining, and cases were then classified negative $(1+$ or $2+)$ or positive $(3+$ or $4+)$. Statistical analysis was performed using correlation of Fisher's Exact test.

Isolation of fecal content and microbiota DNA from feces. Feces were extracted from the colon of WT mice, ressuspended, and homogenized in PBS $1 \times$. The content was centrifuged and the supernatant used for cell stimulation. Fecal DNA was extracted from fresh mouse fecal samples using the QIAamp DNA Stool Mini Kit (QIAGEN, Hilden, Germany).

DNA sequencing of microbiota and bioinformatics workflow. The DNA purified from feces was amplified at the V3-V4 hypervariable region of RNA ribosomal $16 \mathrm{~S}$ and sequenced using the Illumina-MiSeq platform (Illumina, San Diego, CA), which provided single-end reads with $300 \mathrm{nt}$ in length. Downstream analysis were performed by running the QIIME (Quantitative Insights into Microbial Ecology) pipeline. Briefly, sequence reads that presented Phred quality score $<20$ were removed. We normalize each library relative to the smaller sample, choosing randomly until it matches its total number of readings. Afterwards, representative sequences were grouped by Uclust into Operational Taxonomic Units by $97 \%$ similarity and taxonomy assigned with Pynast against Greengenes database. Chimeric sequences were identified by Usearch61 and removed before performing diversity analysis and visualization. The microbiome profile of mice samples was assessed through weighted and unweighted UniFrac principal coordinate analysis. Statistical significance between both groups were inferred by means of the Adonis method implemented in the vegan package for the R statistical software environment, as described before. ${ }^{76}$ The differential abundance of Operational Taxonomic Units in each group was evaluated with the DESeq2 package. We evaluated the alpha diversity in each group by means of the Shannon index. The raw sequence data used in this experiment are available in the EMBL-EBI (Accession id: PRJEB22143).

Lamina propria cell transfection. Lamina propria cells were isolated from WT and STING ${ }^{-1-}$ mice and cultured at $5 \times 10^{5}$ cells per well. Transient transfections of lamina propria cells were carried out using Lipofectamine 2000 (Invitrogen) ratio (in $\mathrm{ml}$ ) of 1:0.25, following the manufacturer's directions. Cells were cultured in RPMI and transfected with microbiota DNA extracted from mice feces ( $3 \mu \mathrm{g}$ per well) or dsDNA90 $\left(3 \mu \mathrm{g} \mathrm{ml}^{-1}\right)$. After $17 \mathrm{~h}$, cells were harvested and assayed for the expression of IFN- $\beta$ by RT-PCR.

Cell culture and nucleic acid transfection. WT and STING ${ }^{-1-}$ MEFs were provided by Dr Glen Barber (University of Miami). Cells were maintained in high-glucose complete Dulbecco's modified Eagle's medium (GIBCO, Waltham, MA) at $37^{\circ} \mathrm{C}$ in $5 \% \mathrm{CO}_{2} / 95 \%$ air in a humidified incubator. MEFs were seeded on 24-well plates containing sterile coverslips a day before the experiment and kept on normal growth media. Cell challenge was performed by DNA transfection using Lipofectamine Reagent (Thermo Fisher Scientific, Waltham, MA) following the manufacturer's instructions. A total of $1 \mu \mathrm{g}$ of DNA extract was used per well. Transfection was performed for $4 \mathrm{~h}$ after which the medium was removed and cells were fixed in $4 \%$

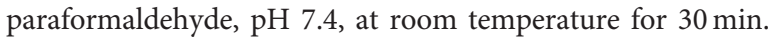

Immunofluorescence and microscopy. After fixation, coverslips were washed with PBS and kept in the same buffer at $4{ }^{\circ} \mathrm{C}$ until immunofluorescence was performed. Permeabilization was carried out for 15 min with $0.3 \%$ Triton X-100 in PBS, and cells were subsequently blocked for $1 \mathrm{~h}$ with $1 \% \mathrm{BSA}$ in $\mathrm{PBS}$ at room temperature prior to incubation with anti-Sting at $4{ }^{\circ} \mathrm{C}$ overnight. Rabbit polyclonal antibody against STING was provided by Glen Barber. ${ }^{77}$ Detection of anti-Sting was performed with anti-rabbit secondary antibody conjugated with Alexa-546 (Jackson Immuno Research, West Grove, PA). Coverslips were mounted in slides using Prolong Gold with 4,6diamidino-2-phenylindole mounting medium (Invitrogen). Microscopy analysis was performed in an Apotome 2.0 (Zeiss, Oberkochen, Germany) system, mounted in a Axio Imager microscope adapted with a Plan-APOCHROMAT $40 \times 0.8$ objective. HXP 120C (metal halide) was used for illumination, and images were acquired as czi files by a AxioCam HRm camera using the Zen software (Zeiss, Oberkochen, Germany).

Cytokine measurements. The colons were homogenized in extraction solution (100 mg tissue per $1 \mathrm{ml}$ ), containing $0.4 \mathrm{M} \mathrm{NaCl}, 0.05 \%$ Tween 20, 0.5\%BSA, 0.1 mM PMSF, $0.1 \mathrm{~mm}$ benzethoniumchloride, $10 \mathrm{~mm}$ EDTA, and $20 \mathrm{KI}$ aprotinin, using Ultra-Turrax. The suspension was then spun at $10,000 \mathrm{~g}$ for $10 \mathrm{~min}$ at $4{ }^{\circ} \mathrm{C}$. The supernatant was used for enzyme-linked immunosorbent assays (ELISAs). Concentrations of IL-4, IL-5, IL-6, IL-10, IL-13, IL-17, and IFN- $\gamma$ were measured in the colon extract by capture ELISA. Concentrations of IL-10 and TGF- $\beta$ were measured in cell culture supernatants and IgA as well as IgM levels were assessed in mice feces by ELISA. ELISAs for cytokine and immunoglobulin (IgA and IgM) measurements were performed using kits from R\&D Systems according to the manufacturer's instructions. For TGF- $\beta$ measurement, samples were acidified and neutralized to activate latent TGF- $\beta$ as previously described. ${ }^{78}$

Statistics. Statistical analysis was performed using GraphPad Prism6 software (GraphPad, La Jolla, CA). Groups of animals were compared using Student's $t$-tests or two-way analysis of variance where applicable. $P$-value $<0.05$ was considered statistically significant.

SUPPLEMENTARY MATERIAL is linked to the online version of the paper at http://www.nature.com/mi

\section{ACKNOWLEDGMENTS}

We thank Daniela Silva dos Reis for help with flow cytometric experiments and Ilda Marçal de Souza and Hermes for the excellent care of the animals. We also thank Tiago Bruno Rezende de Castro for his precious help with figure formatting. We thank Professor André Báfica for his precious contribution to microbiota analysis and for relevant ideas and discussion. This work was supported by scholarships (to M.C.C.C. and T.C.N.) and research fellowships (to A.M.C.F. and S.C.O.) from the Conselho Nacional de Desenvolvimento Científico e Tecnológico (CNPq), Coordenação de Aperfeiçoamento de Pessoal de Nível Superior (CAPES), CAPES/PVE 030448/2013-0, by grants from Fundação de Amparo à Pesquisa de Minas Gerais (FAPEMIG) APQ 00704-14, and National Institute of Health R01 Al116453.

\section{AUTHOR CONTRIBUTIONS}

M.C.C.C., S.C.O., G.N.B., and A.M.C.F. planned experiments and wrote the manuscript. M.C.C.C., L.L., and T.C.N. performed the experiments. J.A. 
performed the assays using MEFs. T.B.R.C. and A.T.V. performed the microbiota analysis. E.S.V., E.F., J.A.-S., and D.C.C. performed histological and imaging analysis. F.M.M. helped with PCR analysis. C.P.Q. and H.C.S. helped with ILC reagents and analysis. F.S.M. provided the germ-free mice and G.N.B. provided STING ${ }^{-1-}$ mice and critical reagents. A.M.C.F., S.C.O., and G.N.B. conceived and supervised the project. All authors provided critical comments.

\section{DISCLOSURE}

The authors declared no conflict of interest.

c 2018 Society for Mucosal Immunology

\section{REFERENCES}

1. Mayer, L. Mucosal immunity. Immunol. Rev. 206, 5 (2005).

2. Weiner, H.L., da Cunha, A.P., Quintana, F. \& Wu, H. Oral tolerance. Immunol. Rev. 241, 241-259 (2011).

3. Pabst, O. \& Mowat, A.M. Oral tolerance to food protein. Mucosal Immunol. 5, 232-239 (2012).

4. Honda, K. \& Littman, D.R. The microbiome in infectious disease and inflammation. Annu. Rev. Immunol. 30, 759-795 (2012).

5. Hill, D.A. \& Artis, D. Intestinal bacteria and the regulation of immune cell homeostasis. Annu. Rev. Immunol. 28, 623-667 (2010).

6. Crosnier, C., Stamataki, D. \& Lewis, J. Organizing cell renewal in the intestine: stem cells, signals and combinatorial control. Nat. Rev. Genet. 7, 349-359 (2006).

7. Hu, S. et al. The DNA sensor AIM2 maintains intestinal homeostasis via regulation of epithelial antimicrobial host defense. Cell Rep. 13, 1922-1936 (2015).

8. Fukata, M. \& Arditi, M. The role of pattern recognition receptors in intestinal inflammation. Mucosal Immunol. 6, 451-463 (2013).

9. Rakoff-Nahoum, S., Paglino, J., Eslami-Varzaneh, F., Edberg, S. \& Medzhitov, R. Recognition of commensal microflora by toll-like receptors is required for intestinal homeostasis. Cell 118, 229-241 (2004).

10. Hu, G.Q. et al. AlM2 contributes to the maintenance of intestinal integrity via Akt and protects against Salmonella mucosal infection. Mucosal Immunol. 9, 1330-1339 (2016).

11. Ishikawa, H. \& Barber, G.N. STING is an endoplasmic reticulum adaptor that facilitates innate immune signalling. Nature 455, 674-678 (2008).

12. Burdette, D.L. et al. STING is a direct innate immune sensor of cyclic diGMP. Nature 478, 515-518 (2011).

13. Sun, L., Wu, J., Du, F., Chen, X. \& Chen, Z.J. Cyclic GMP-AMP synthase is a cytosolic DNA sensor that activates the type I interferon pathway. Science 339, 786-791 (2013).

14. Burdette, D.L. \& Vance, R.E. STING and the innate immune response to nucleic acids in the cytosol. Nat. Immunol. 14, 19-26 (2013).

15. Zhong, B. et al. The adaptor protein MITA links virus-sensing receptors to IRF3 transcription factor activation. Immunity 29, 538-550 (2008).

16. Sun, W. et al. ERIS, an endoplasmic reticulum IFN stimulator, activates innate immune signaling through dimerization. Proc. Natl. Acad. Sci. USA 106, 8653-8658 (2009).

17. Jin, L., Waterman, P.M., Jonscher, K.R., Short, C.M., Reisdorph, N.A. \& Cambier, J.C. MPYS, a novel membrane tetraspanner, is associated with major histocompatibility complex class II and mediates transduction of apoptotic signals. Mol. Cell Biol. 28, 5014-5026 (2008).

18. Cho, H. \& Kelsall, B.L. The role of type I interferons in intestinal infection, homeostasis, and inflammation. Immunol. Rev. 260, 145-167 (2014).

19. Chirdo, F.G., Millington, O.R., Beacock-Sharp, H. \& Mowat, A.M. Immunomodulatory dendritic cells in intestinal lamina propria. Eur. J. Immunol. 35, 1831-1840 (2005).

20. Kole, A. et al. Type I IFNs regulate effector and regulatory $T$ cell accumulation and anti-inflammatory cytokine production during $T$ cellmediated colitis. J. Immunol. 191, 2771-2779 (2013).

21. Sauer, J.D. et al. The N-ethyl-N-nitrosourea-induced Goldenticket mouse mutant reveals an essential function of Sting in the in vivo interferon response to Listeria monocytogenes and cyclic dinucleotides. Infect. Immun. 79, 688-694 (2011).

22. Dobbs, N., Burnaevskiy, N., Chen, D., Gonugunta, V.K., Alto, N.M. \& Yan, N. STING activation by translocation from the ER is associated with infection and autoinflammatory disease. Cell Host Microbe 18, 157-168 (2015).

23. Zhu, Q. et al. Cutting edge: STING mediates protection against colorectal tumorigenesis by governing the magnitude of intestinal inflammation. $J$. Immunol. 193, 4779-4782 (2014).

24. Ahn, J., Konno, H. \& Barber, G.N. Diverse roles of STINGdependent signaling on the development of cancer. Oncogene 34, 5302-5308 (2015).

25. Rudlaff, R.M. \& Waters, C.M. What is the role of cyclic di-GMP signaling within the human gut microbiome? . Microbiome Sci. Med. 1, 39-44 (2014).

26. Danilchanka, O. \& Mekalanos, J.J. Cyclic dinucleotides and the innate immune response. Cell 154, 962-970 (2013).

27. Hooper, L.V., Littman, D.R. \& Macpherson, A.J. Interactions between the microbiota and the immune system. Science 336, 1268-1273 (2012).

28. Nowacki, M.R. Cell proliferation in colonic crypts of germ-free and conventional mice-preliminary report. Folia Histochem. Cytobiol. 31, 7781 (1993).

29. Abrams, G.D., Bauer, H. \& Sprinz, H. Influence of the normal flora on mucosal morphology and cellular renewal in the ileum. A comparison of germ-free and conventional mice. Lab. Invest. 12, 355-364 (1963).

30. Petersson, J. et al. Importance and regulation of the colonic mucus barrier in a mouse model of colitis. Am. J. Physiol. Gastrointest. Liver Physiol. 300, G327-G333 (2011).

31. Round, J.L. \& Mazmanian, S.K. The gut microbiota shapes intestinal immune responses during health and disease. Nat. Rev. Immunol. 9, 313323 (2009).

32. Hapfelmeier, S. et al. Reversible microbial colonization of germ-free mice reveals the dynamics of IgA immune responses. Science 328, 1705-1709 (2010).

33. Benveniste, J., Lespinats, G. \& Salomon, J. Serum and secretory IgA in axenic and holoxenic mice. J. Immunol. 107, 1656-1662 (1971).

34. Suzuki, K. et al. Aberrant expansion of segmented filamentous bacteria in IgA-deficient gut. Proc. Natl. Acad. Sci. USA 101, 1981-1986 (2004).

35. Fransen, F. et al. BALB/c and C57BL/6 mice differ in polyreactive IgA abundance, which impacts the generation of antigen-specific IgA and microbiota diversity. Immunity 43, 527-540 (2015).

36. Rowan, F., Docherty, N.G., Murphy, M., Murphy, B., Calvin Coffey, J. \& O'Connell, P.R. Desulfovibrio bacterial species are increased in ulcerative colitis. Dis. Colon Rectum 53, 1530-1536 (2010).

37. Smith, P.M. et al. The microbial metabolites, short-chain fatty acids, regulate colonic Treg cell homeostasis. Science 341, 569-573 (2013).

38. Ren, W. et al. Interferon Tau affects mouse intestinal microbiota and expression of IL-17. Mediat. Inflamm. 2016, 2839232 (2016).

39. Umesaki, Y., Setoyama, H., Matsumoto, S. \& Okada, Y. Expansion of alpha beta T-cell receptor-bearing intestinal intraepithelial lymphocytes after microbial colonization in germ-free mice and its independence from thymus. Immunology 79, 32-37 (1993).

40. Cheroutre, H., Lambolez, F. \& Mucida, D. The light and dark sides of intestinal intraepithelial lymphocytes. Nat. Rev. Immunol. 11, 445-456 (2011).

41. Klose, C.S. \& Artis, D. Innate lymphoid cells as regulators of immunity, inflammation and tissue homeostasis. Nat. Immunol. 17, 765-774 (2016).

42. Diefenbach, A., Colonna, M. \& Koyasu, S. Development, differentiation, and diversity of innate lymphoid cells. Immunity 41, 354-365 (2014).

43. Chen, M.L., Yan, B.S., Bando, Y., Kuchroo, V.K. \& Weiner, H.L. Latencyassociated peptide identifies a novel CD4 $+\mathrm{CD} 25+$ regulatory $\mathrm{T}$ cell subset with TGFbeta-mediated function and enhanced suppression of experimental autoimmune encephalomyelitis. J. Immunol. 180, 73277337 (2008)

44. Bilate, A.M. \& Lafaille, J.J. Induced CD4 + Foxp3 + regulatory T cells in immune tolerance. Annu. Rev. Immunol. 30, 733-758 (2012).

45. Metidji, A., Rieder, S.A., Glass, D.D., Cremer, I., Punkosdy, G.A. \& Shevach, E.M. IFN-alpha/beta receptor signaling promotes regulatory $T$ cell development and function under stress conditions. J. Immunol. 194, 4265-4276 (2015).

46. Lee, S.E. et al. Type I interferons maintain Foxp3 expression and T-regulatory cell functions under inflammatory conditions in mice. Gastroenterology 143, 145-154 (2012).

47. Kiesler, P., Fuss, I.J. \& Strober, W. Experimental models of inflammatory bowel diseases. Cell. Mol. Gastroenterol. Hepatol. 1, 154-170 (2015). 
48. Leach, M.W., Bean, A.G., Mauze, S., Coffman, R.L. \& Powrie, F. Inflammatory bowel disease in C.B-17 scid mice reconstituted with the CD45RBhigh subset of CD4 + T cells. Am. J. Pathol. 148, 1503-1515 (1996).

49. Katakura, K., Lee, J., Rachmilewitz, D., Li, G., Eckmann, L. \& Raz, E. Tolllike receptor 9-induced type IIFN protects mice from experimental colitis. J. Clin. Invest. 115, 695-702 (2005).

50. Rachmilewitz, D. et al. Immunostimulatory DNA ameliorates experimental and spontaneous murine colitis. Gastroenterology 122, 1428-1441 (2002).

51. Tschurtschenthaler, M. et al. Type I interferon signalling in the intestinal epithelium affects Paneth cells, microbial ecology and epithelial regeneration. Gut 63, 1921-1931 (2014).

52. Bos, N.A., Meeuwsen, C.G., Wostmann, B.S., Pleasants, J.R. \& Benner, R. The influence of exogenous antigenic stimulation on the specificity repertoire of background immunoglobulin-secreting cells of different isotypes. Cell. Immunol. 112, 371-380.

53. Pabst, O., Cerovic, V. \& Hornef, M. Secretory IgA in the coordination of establishment and maintenance of the microbiota. Trends Immunol. 37, 287-296 (2016).

54. Berry, D. \& Reinisch, W. Intestinal microbiota: a source of novel biomarkers in inflammatory bowel diseases?. Best Pract. Res. Clin. Gastroenterol. 27, 47-58 (2013).

55. Dong, P., Yang, Y. \& Wang, W.P. The role of intestinal bifidobacteria on immune system development in young rats. Early Hum. Dev. 86, 51-58 (2010).

56. Hoytema van Konijnenburg, D.P. \& Mucida, D. Intraepithelial lymphocytes. Curr. Biol. 27, R737-R739 (2017).

57. Melo-Gonzalez, F. \& Hepworth, M.R. Functional and phenotypic heterogeneity of group 3 innate lymphoid cells. Immunology 150, 265-275 (2017).

58. Monticelli, L.A., Osborne, L.C., Noti, M., Tran, S.V., Zaiss, D.M. \& Artis, D. IL-33 promotes an innate immune pathway of intestinal tissue protection dependent on amphiregulin-EGFR interactions. Proc. Natl. Acad. Sci. USA 112, 10762-10767 (2015).

59. Negishi, H. et al. Essential contribution of IRF3 to intestinal homeostasis and microbiota-mediated Tslp gene induction. Proc. Natl. Acad. Sci. USA 109, 21016-21021 (2012).

60. Song, C. et al. Unique and redundant functions of NKp46 + ILC3s in models of intestinal inflammation. J. Exp. Med. 212, 1869-1882 (2015).

61. Buonocore, S. et al. Innate lymphoid cells drive interleukin-23-dependent innate intestinal pathology. Nature 464, 1371-1375 (2010).

62. Bernink, J.H. et al. Human type 1 innate lymphoid cells accumulate in inflamed mucosal tissues. Nat. Immunol. 14, 221-229 (2013).

63. Izcue, A., Coombes, J.L. \& Powrie, F. Regulatory Tcells suppress systemic and mucosal immune activation to control intestinal inflammation. Immunol. Rev. 212, 256-271 (2006).
64. Sun, M., He, C., Cong, Y. \& Liu, Z. Regulatory immune cells in regulation of intestinal inflammatory response to microbiota. Mucosal Immunol. 8, 969978 (2015).

65. Faria, A.M.C., Reis, B.S. \& Mucida, D. Tissue adaptation: Implications for gut immunity and tolerance. J. Exp. Med. 214, 1211-1226 (2017).

66. Mowat, A.M. \& Agace, W.W. Regional specialization within the intestinal immune system. Nat. Rev. Immunol. 14, 667-685 (2014).

67. Ishinaga, H., Takeuchi, K., Kishioka, C., Suzuki, S., Basbaum, C. \& Majima, Y. Pranlukast inhibits NF-kappaB activation and MUC2 gene expression in cultured human epithelial cells. Pharmacology 73, 89-96 (2005).

68. Ahn, D.H. et al. TNF-alpha activates MUC2 transcription via NFkappaB but inhibits via JNK activation. Cell. Physiol. Biochem. 15, 29-40 (2005).

69. Jono, H. et al. Transforming growth factor-beta -Smad signaling pathway cooperates with NF-kappa B to mediate nontypeable Haemophilus influenzae-induced MUC2 mucin transcription. J. Biol. Chem. 277, 45547-45557 (2002).

70. Shan, M. et al. Mucus enhances gut homeostasis and oral tolerance by delivering immunoregulatory signals. Science 342, 447-453 (2013).

71. Velcich, A. et al. Colorectal cancer in mice genetically deficient in the mucin Muc2. Science 295, 1726-1729 (2002).

72. Kranzusch, P.J., Wilson, S.C., Lee, A.S., Berger, J.M., Doudna, J.A. \& Vance, R.E. Ancient origin of CGAS-STING reveals mechanism of universal 2',3' cGAMP signaling. Mol. Cell 59, 891-903 (2015).

73. Romling, U. Great times for small molecules: c-di-AMP, a second messenger candidate in Bacteria and Archaea. Sci. Signal. 1, pe39 (2008).

74. Zhang, X. et al. Cyclic GMP-AMP containing mixed phosphodiester linkages is an endogenous high-affinity ligand for STING. Mol. Cell 51, 226235 (2013).

75. de Almeida, L.A. et al. MyD88 and STING signaling pathways are required for IRF3-mediated IFN-beta induction in response to Brucella abortus infection. PLOS ONE 6, e23135 (2011).

76. Powrie, F., Leach, M.W., Mauze, S., Menon, S., Caddle, L.B. \& Coffman, R.L. Inhibition of Th1 responses prevents inflammatory bowel disease in scid mice reconstituted with CD45RBhi CD4 + T cells. Immunity 1, 553-562 (1994).

77. Marino, E. et al. Gut microbial metabolites limit the frequency of autoimmune T cells and protect against type 1 diabetes. Nat. Immunol. 18, 552-562 (2017).

78. Faria, A.M., Maron, R., Ficker, S.M., Slavin, A.J., Spahn, T. \& Weiner, H.L. Oral tolerance induced by continuous feeding: enhanced up-regulation of transforming growth factor-beta/interleukin-10 and suppression of experimental autoimmune encephalomyelitis. J. Autoimmun. 20, 135-145 (2003). 